\title{
L'état actuel de la technique des conduites forcées souterraines
}

\section{The present state of underground penstock technique}

\author{
PAR M. Joseph TALOBRE
}

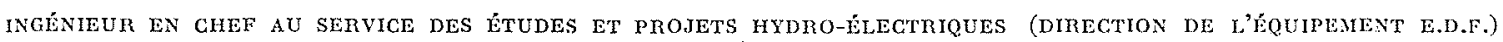

\begin{abstract}
Divers types de conduites forcées souterraines. - Les caractéristiques mécaniques des roches, leur hétérogénéité, leur compression náturelle. - Mesures de compressibilité et détermination des contraintes à l'intérieur du rocher. - Recommandations pour l'exécution des conduites forcées souterraines. - Les lecons de l'expérience et des accidents survenus sur certains puits de chute.
\end{abstract}

Les conduites forcées, par lesquelles le débit transporté par les galeries d'amenée parvient aux turbines des usines hydro-électriques, sont beaucoup plus souvent qu'autrefois prévues souterraines. Cela pour les raisons qui font adopter les usines souterraines, à savoir la protection d'un site, une protection militaire, des nécessités topographiques, mais le plus souvent des raisons d'économie de métal et de capitaux. Il est évident que les progrès de la technique ont rendu de tels ouvrages souterrains chaque jour plus réalisables et plus sûrs. Les procédés de construction se sont perfectionnés. Le prix des déblais en souterrain a considérablement diminué, en même temps que diminuaient les délais et les aléas de la perforation. Enfin les éléments du calcul de ces ouvrages sont chaque jour mieux connus.

\section{Corstitution \\ d'une conduite forcée souterraine}

Une conduite forcée souterraine est spécifiquement constituée d'un blindage en acier bloqué par du béton dans une excavation en rocher. Mais ce type d'ouvrage est loin de se présenter sous une forme unique et définitive. D'ailleurs toute technique évolue, et la technique des conduites forcées souterraines n'a pas fait exception à cette règle. Le meilleur moyen de s'en rendre compte est de passer rapidement en revue les principales réalisations de ces trente dernières années.

\section{Condutes a blindage épais.}

Dans un premier groupe, qui est le plus ancien et le plus étendu, le blindage est l'organe principal de la résistance. Souvent, il a été étudié comme une transposition de la conduite forcée aérienne, compte tenu seulement d'une certaine
The various types of underground penstocks. Mechanical characteristics of rocks, their heterogeneity and their internal stresses. Compres. sibility measurements and determination of stresses within rock. Recommendations concerning the construction of underground penstocks. The lessons derived from experience and from accidents occured in some underground penstocks.

adaptation à sa position souterraine. Un intermédiaire assez fréquent entre la conduite blindée souterraine et la conduite forcée ordinaire est d'ailleurs la conduite forcée posée en galerie, qui ne demande au rocher qu'un passage plus direct, ou qu'une protection contre les avalanches.

La conduite épaisse bloquée en rocher, par une sorte d'analogie avec ce dernier type, est en général calculée comme un tuyau libre, avec toutefois un taux de fatigue plus élevé pour le métal. La seule condition requise est le plus souvent d'éviter les contraintes égales ou supérieures à la limite d'élasticité de la tôle. Le rocher et le béton par contre ne font l'objet que de contrôles généraux, leur rôle reconnu étant de fournir un appoint de résistance, utile, mais non indispensable et pouvant rester de ce fait quelque peu aléatoire.

Si l'on examine des cas concrets, on trouvera par exemple un taux de travail théorique, d'environ $16 \mathrm{~kg} / \mathrm{mm}^{2}$ dans les tôles de 12 à $24 \mathrm{~mm}$ du blindage de la conduite de Brommat, de $18 \mathrm{~kg} / \mathrm{mm}^{2}$ à la Gerlos, de $24 \mathrm{~kg} / \mathrm{mm}^{2}$ à Péage de Vizille, de $27 \mathrm{~kg} / \mathrm{mm}^{2}$ à Handeck. Les conduites de Brommat et d'Handeck sont dans du granit. Cela revient à escompter en général que la participation normale de la roche à la résistance égalera à peu près celle de la tôle. Toutefois, et depuis un quart de siècle au moins, des tentatives ont été faites pour préciser cette proportion. Des auteurs, parmi lesquels nous citerons au hasard Kochlin, Marllart, Hoffmann, Hutter, KastNER, ont fait jouer les élasticités relatives des anneaux d'acier, de béton et de rocher pour calculer la répartition des efforts, et la part du blindage. Il a même été tenu compte des vides de quelques dixièmes de millimètres au joint acierbéton et au joint béton-rocher, ainsi que de l'influence des contractions d'origine thermique.

Pour contrôler les hypothèses faites, des essais 


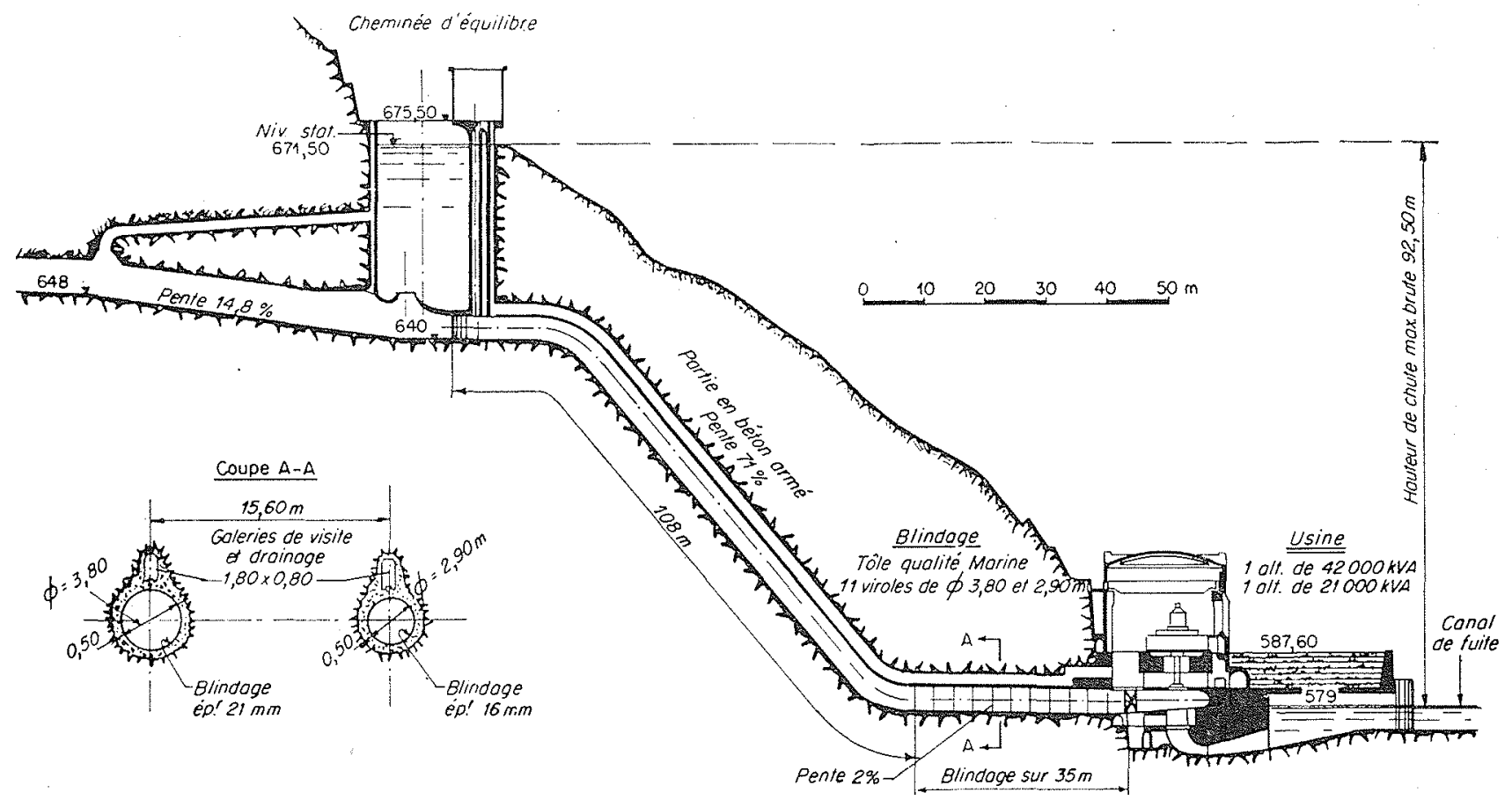

Fig. 1. - Profil en long de la conduite souterrate de Cordéac.

en vraie grandeur ont été parfois poursuivis sur des cavernes revêtues ou sur des tronçons, notamment à Amsteg, Lucendro, Malgovert, Lyse, Lovero, Santa-Giustina ou Castelbello, dont on a mesuré les dilatations diamétrales sous l'effet d'une pression intérieure variable. Nous en reparlerons ultérieurement.

La résistance des blindages aux pressions extérieures, ou sous-pressions, n'a d'ailleurs pas toujours été assez certaine pour permettre une réduction très poussée des épaisseurs de tôle. On a craint que des fuites ne viennent alimenter les nappes naturelles, et augmentent leur pression qui est souvent de $10 \mathrm{~kg} / \mathrm{cm}^{2}$ ou plus. L'ancrage des tôles n'a été que très exceptionnellement envisagé et on a généralement préféré drainer le béton de blocage des conduites. Comme les drains de petit diamètre se bouchaient à ]a longue, on a voulu rendre ces drains permanents et visitables en leur donnant une grande section. Tel est le cas de Mera, Regoledo, Santa-Massenza, Soverzene, Castelbello, Cordéac (fig. 1), Brévières et la Gerlos (fig. 2). Les conduites de Castelbello et de Cordéac sont d'ailleurs en béton armé et les galeries servent surtout à la surveillance.

Les essais photo-élasticimétriques effectués pour la conduite des Brévières ont prouvé que de telles galeries de drainage amenent dans le béton de revêtement des majorations importantes de contraintes. D'autre part si leur utilité reste incertaine, et si leur accessibilité apparaît très théorique, la dissymétrie qu'elles entrainent pour la section d'excavation, qui s'éloigne de la forme circulaire idéale, est trop évidente. Aussi faut-il noter que de telles galeries n'ont été prévues ni à Montpezat, chute actuellement en achèvement, ni à Pfaffensprung, Albigna, Innertkirchen ou Handeck.

Cette question des galeries de drainage sera discutée d'ailleurs par la suite.

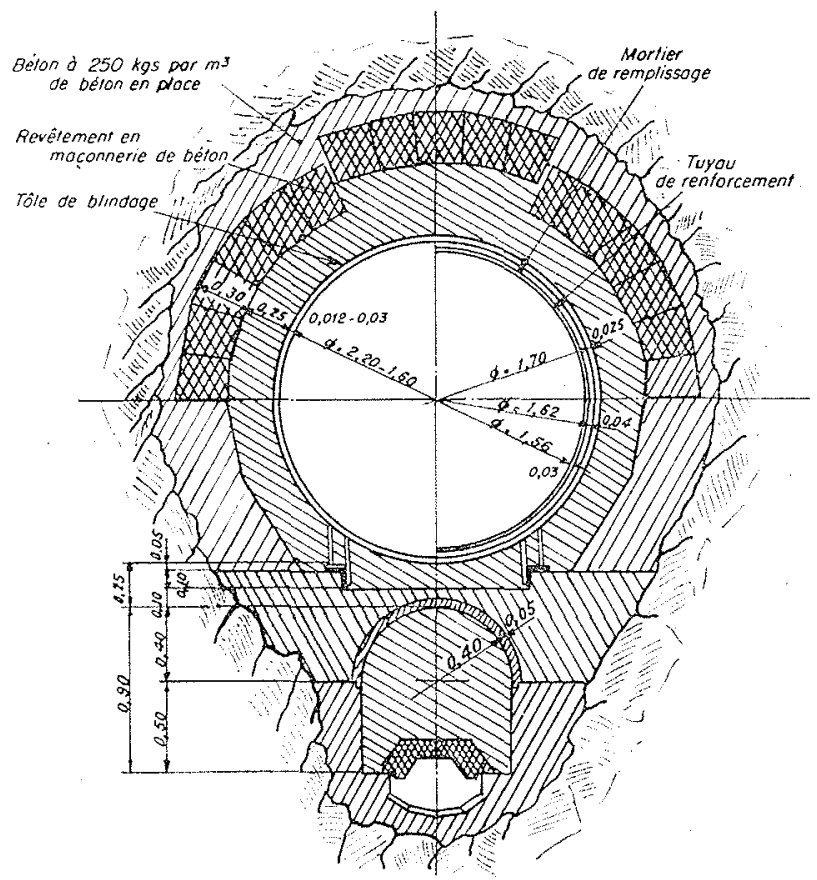

Fig. 2. - Chute de ta Gerlos. Coupe transversale de la conduite blindée. 


\section{LIES BLINDAGES SERRÉS PAR INJECTION.}

Un important progrès dont les conséquences n'ont pas été vues dès le début résulte de l'emploi du serrage des bétons de blocage par injection de ciment. Ce serrage permet la suppression des galeries de drainage, du fait que la circulation des eaux extérieures est considérablement freinée, et aussi du fait que la résistance au flambage de la tôle est substantiellement accrue. Il supprime presque totalement les vides au contact des divers matériaux. Mais surtout, il constitue une première réalisation de la mise en compression préalable du blindage et du béton, c'est-à-dire une application du principe de la précontrainte. Si l'injection pouvait être menée avec un plein succès dans tous les cas, ce qui n'est pas avec les techniques actuelles trop irrégulières, il serait donc possible d'imposer au rocher la charge maximum qu'il peut supporter, et le blindage pourrait donc être allégé considérablement.

Souvent d'ailleurs on s'apercevrait que l'acier n'est pas indispensable à la résistance et que son utilité est surtout de protéger le rocher trop fissuré pour être étanche ou trop attaquable par l'eau.

\section{LF CHEMISAGE SOUPLE MARINONI.}

Ce fut le mérite de Marrnoni de réaliser dès 1925, avec une hardiesse extrême pour l'époque, des conduites forcées souterraines de 527,720 et 758 mètres de charge statique, à Pallanzeno,

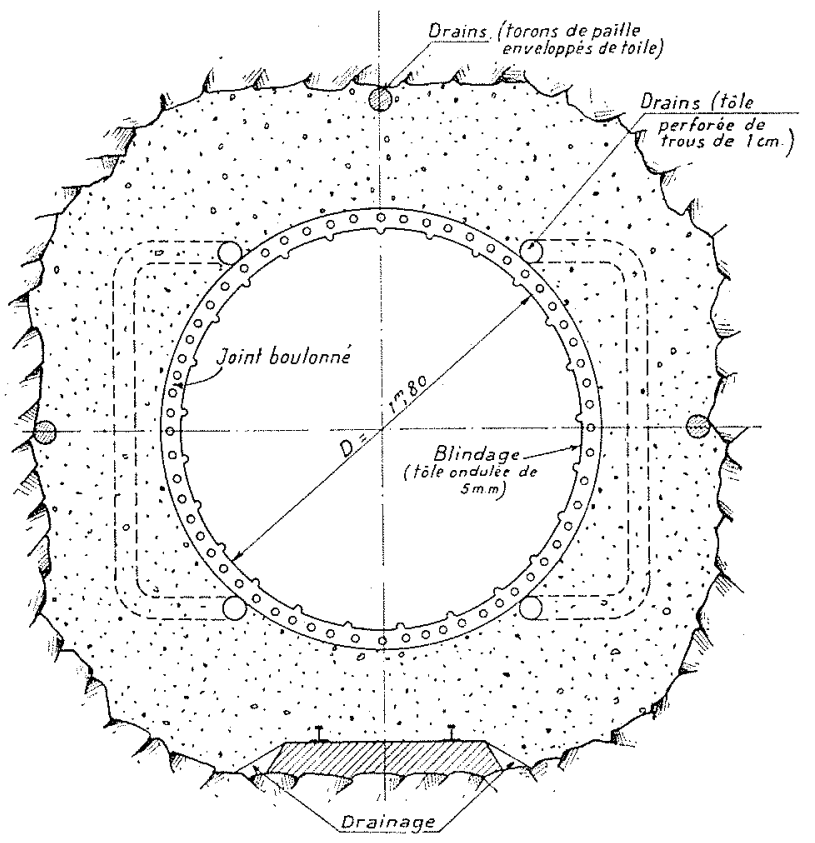

HAUTEUR OE CHUTE MAX. BRUTE. $758 \mathrm{~m}$.

Fig. 3. - Coupe du puts de chute de Mese.
Rovesca et Mese (fig. 3), conduites dont le chemisage souple ne dépassait pas $4 \mathrm{~mm}$ d'épaisseur. Les terrains traversés n'étaient pas des plus compacts, et les tracés étaient souvent maintenus dangereusement à flanc de versant. Malgré le défaut de couverture, les accidents, après un quart de siècle d'exploitation de ces conduites, se révèlent dus surtout aux sous-pressions. MaRINONI, en effet, avait voulu que le chemisage d'acier fut visitable. Peut-être eut-il tort sur ce point. Mais l'expérience des nombreuses conduites du type Marinon prouve du moins que les blindages ne sont pas indispensables à la stabilité de ces ouvrages, ceux-ci fussent-ils à très forte charge.

\section{LES REVETEMENTS EN BÉTON.}

Les récents progrès accomplis grâce à la précontrainte des bétons en ce qui concerne le revètement des galeries d'amenée devaient nécessairement avoir une influence sur le dessin des conduites forcées souterraines à charge moyenne. Un revêtement de béton de granulométrie étudiée reste suffisamment étanche aux fortes charges tant qu'il obéit à la règle de Maurice Lévy, c'esta-dire tant que sa compression est supérieure à la pression hydraulique. C'est bien ce que l'on peut constater sur les conduites aériennes en béton fretté et par exemple sur les conduites forcées de la Selve. On concoit, toutefois, que les pressions que peut supporter un revêtement bétonné soient limitées. En pratique, on pourra difficilement dépasser une charge de 200 mètres, qui demande un excédent de compression de 30 à $40 \mathrm{~kg} / \mathrm{cm}^{2}$ pour l'étanchéité du béton. Bien que limité, le champ d'application du revêtement précontraint pour les puits de chute et les conduites souterraines reste cependant important, et dès à présent on peut citer des ouvrages remarquables de ce type. Un des premiers exemples, sinon le premier, de précontrainte appliquée à une conduite forcée souterraine est celui de Marèges, où le serrage du béton a été obtenu par la mise en tension préalable d'armatures selon un procédé imaginé par M. Mary. Plus récemment, M. Freyssiner a utilisé aussi la précontrainte du béton par fils pour la traversée sous-fluviale de La Frette. La précontrainte a d'ailleurs été utilisée aussi hor's de France; notamment à Soverzene, avec une conduite souterraine double, et pour une chute brute de 210 mètres. A Muccone, une galerie de $2,70 \mathrm{~m}$ de diamètre intérieur supporte une charge totale de 120 mètres. (fig. 4). La paroi d'étanchéité en béton préfabriqué sous vide, , de $12 \mathrm{~cm}$ d'épaisseur, est frettée par du fil d'acier à haute résistance. Un espace annulaire de $55 \mathrm{~mm}$ de largeur est laissé au pourtour du tube précontraint pour le blocage, effectué au mortier sous pression. Toutefois, ce système ne pourrait guère être 
étendu aux très grands diamètres, étant donné le poids des viroles.

La mise en tension des fils entraine d'ailleurs, une complication certaine dans l'exécution et on a cherché à s'en libérer par la mise en précon-

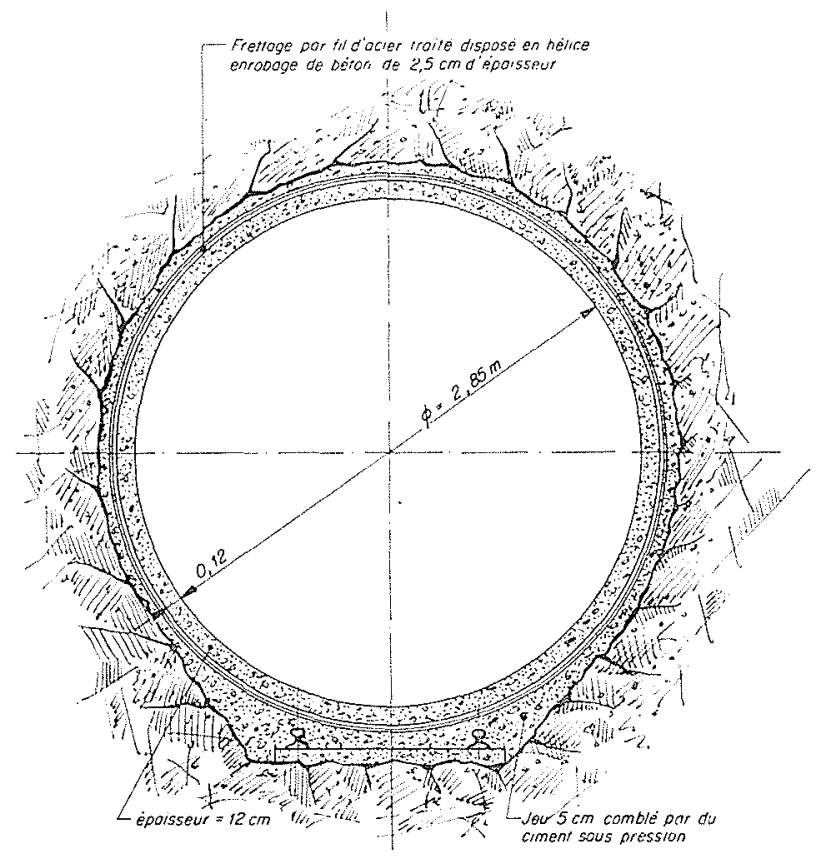

Frg. 4. - Galenie en charge bétonnée frettée DE MuCCONE.

traite par injection seulement. Pour égaliser l'effet de l'injection, il est nécessaire de prévoir, au pourtour de l'anneau de béton à précontraindre, un espace annulaire suffisant. Dès 1942, la précontrainte par injection dans un espace annulaire de $10 \mathrm{~cm}$ de largeur était appliquée à Eylie, pour le renforcement local d'une galerie d'amenée fonctionnant sous 40 mètres de charge (fig. 5). La même idée a été reprise en Autriche et perfectionnée par M. Kreser. M. Freyssnnet a appliqué sur une grande échelle ses procédés à d'importants ouvrages d'Afrique du Nord : DjenDjen, Oued-Agrioun, Ben-Métir, Oued-Fodda. Dans les réalisations récentes, le vide annulaire est réduit à 4 ou $5 \mathrm{~cm}$. Le remplissage en est fait par des mortiers spéciaux injectés à une pression suffisante pour réaliser tant l'essorage, en vue d'éliminer l'excédent d'eau nuisible à la résistance, que le maintien du serrage. Les relaxations constatées ont souvent été inférieures à $30 \%$ de la pression d'injection.

Le béton armé ordinaire a été aussi ntilisé. Un tel béton ne peut supporter de traction supérieure a 25 ou $30 \mathrm{~kg} / \mathrm{cm}^{2}$ sans fissuration, donc sans perte d'étanchéité. L'armature simple ne donne aucune sécurité d'étanchéité en mauvais terrain et lon devra limiter son emploi aux con- duites en bon rocher et à faible pression. Toutefois, on peut citer des conduites souterraines en béton armé donnant toule satisfaction, et par exemple les conduites de Cordéac, de Moux, de Castelbello et de Bressanone. A Castelbello, sous 294,70 m de chute brute, l'étanchéité a été obtenue parfaitement avec une armature grillagée fine et très serrée recouvrant l'anneau porteur de béton, armé à $200 \mathrm{~kg}$ de fer par mètre eube, et de $50 \mathrm{~cm}$ d'épaisseur. Le produit du diamètre par la pression de service atteint ici le chiffre record pour les conduites en béton de 830 tonnes-mètre. Bien entendu, les revêtements en béton sont pratiquement insensibles aux souspressions, et ce n'est pas là leur moindre avantage.

\section{Puits de chute NoN REVÊTUS.}

Tout revêtement peut même être supprimé lorsque le rocher est indécomposable à l'eau, lor'squ'il est stable, et lorsqu'il est suffisamment étanche. C'est la solution qui a été admise à Svelgen (Norvège) dès 1920 pour une chute de 152 mètres, et à Salarno (Italie), pour une chute de $162 \mathrm{~m}$. La suppression de tout revêtement avait été envisagée pour la chute du Cañon del. Pato, au Pérou, de 420 mètres. Malheureusement

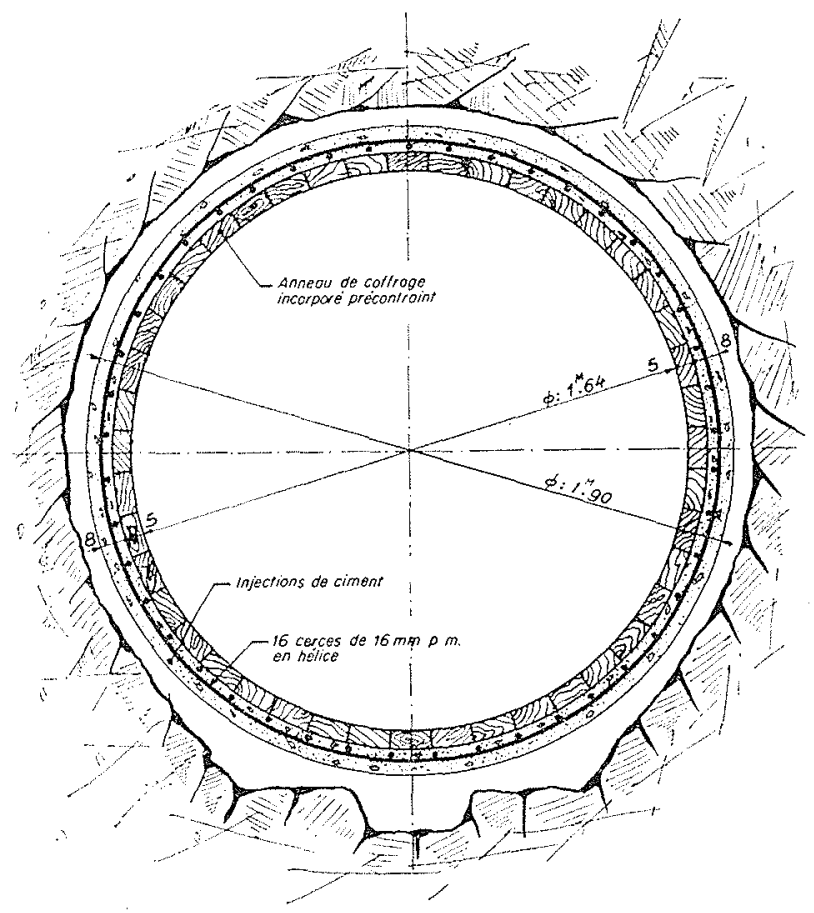

Fig. 5. - Coupe de la gaterie d'amenée d'Eylie (charge $40 \mathrm{~m}$ ).

le granite s'est révélé recoupé par des cassures pouvant constituer un risque sérieux pour l'étanchéité. Un chemisage a été reconnu nécessaire.

Les conduites forcées souterraines ne sont 
donc pas nécessairement des conduites métalliques enrobées de béton, et il est même parfois difficile de les séparer des galeries d'amenée à forte charge dont elles empruntent les technicques.

Des trois matériaux constitutifs de la conduite souterraine, l'acier, le béton et le rocher, les deux premiers peuvent parfois faire défaut. Le premier surtout, le dernier jamais. C'est done par ce dernier, le rocher, que nous commencerons notre examen des propriétés des matériaux des conduites D'autant plus que le rocher, malgré son rôle primordial, reste encore fort mal connu.

\section{Les roches et leurs propriétés mécaniques}

Les roches sont essentiellement hétérogènes. A l'échelle microscopique d'abord, puisqu'elles sont formées de l'assemblage de cristaux de natures diverses. A l'échelle métrique ensuite, car elles présentent des accidents, qui sont les cli-

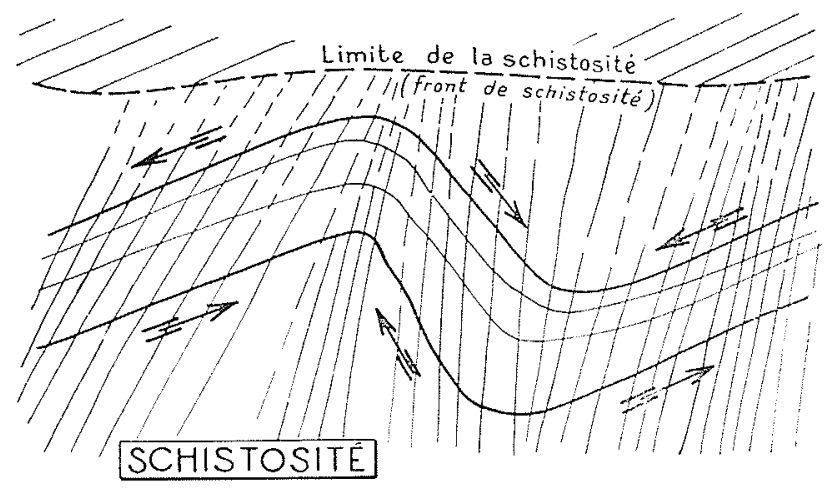

FIG. 6.

vages dus à l'histoire extrêmement complexe do la roche. Les clivages comprennent la stratification, les diaclases et failles et la schistosité (fig. 6). En outre, les roches peuvent être affectées, plus ou moins profondément, par le métamorphisme.

\section{Propriétés DES ROCHES.}

Les principales propriétés des roches qui intéressent la construction des conduites et puits de chute, et que l'on doit mesurer sur place, sont la résistance de la roche aux charges qui lui sont applicquées, sa déformabilité et son étanchéité.

\section{Résistance à la compression}

La résistance de la roche à la rupture sous compression mesurée sur échantillons dépourvus de fissures, serrés normalement au litage est généralement très élevée.

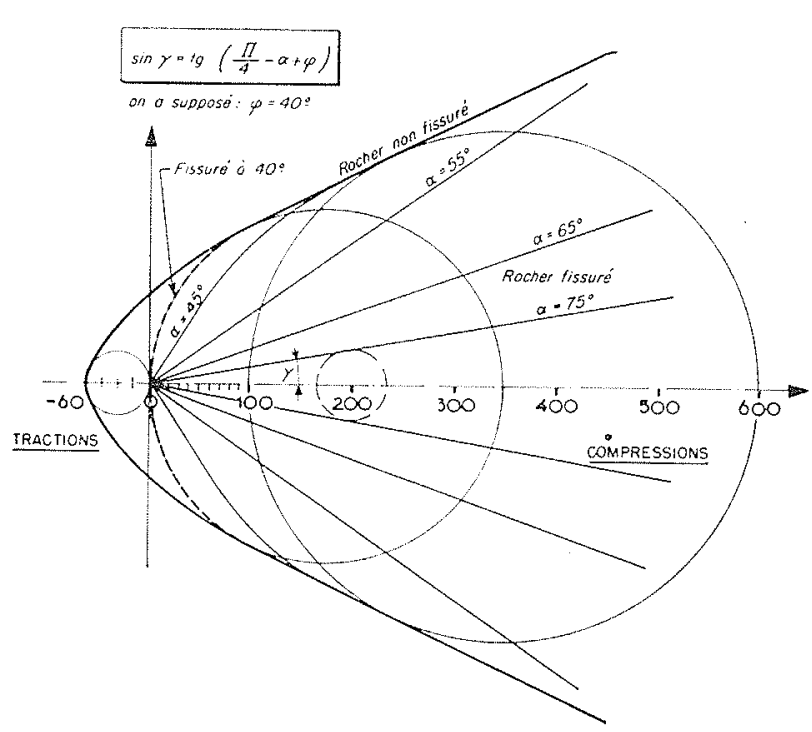

Fig. 7. - Coupe intrinsèQue du rocher fissuré.

On augmente considérablement cette résistance à l'écrasement par une compression latérale ou étreinte. De nombreux essais avec étreinte transversale ont été effectués sur des pierres. Si l'on porte sur un axe les tensions prin cipales extrêmes et si l'on construit les cercles de Morr correspondants, leur enveloppe est une courbe de résistance intrinsèque analogue à celle obtenue pour les bétons (fig. 7).

Si la roche est découpée du fait du clivage, les résultats obtenus ne coïncident avec ceux de l'échantillon compact, comprimé suivant les lits, que si l'angle du clivage avec la direction des forces est supérieur à $\pi / 2$ ment de la roche sur elle-même étant o. Mais si l'éprouvette essayée est soumise à étreinte, cet angle peut être beaucoup diminué (fig. 8). L'application de la règle de Coulonis permet en outre de prévoir la modification que subira la courbe de résistance intrinsèque.

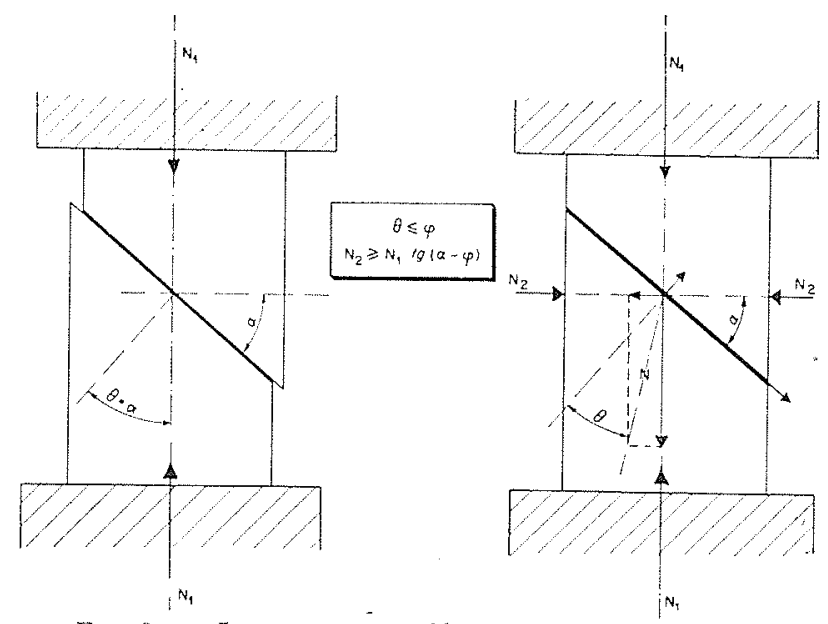

Fig. 8. - Influence de l'útrente transversale. 
Toute roche étant découpée par plusieurs systèmes diversement inclinés de cassures, on concoit que sa résistance intrinsèçue soit essentiellement variable suivant les diverses directions de l'espace. On peut donc fortement mettre en doute les théories qui s'appuient sur l'hypothèse de l'homogénéité et de l'isotropie du terrain. Toutefois en pratique, la roche fissurée se comporte, étant donné sa compression naturelle en triple étreinte, comme un véritable solide. Mais ce n'est qu'avec une certaine réserve qu'il est permis à la théorie de l'élasticité d'intervenir, étant donné les anisotropies et compte tenu des tensions internes acquises antérieurement par le massif.

\section{Compression naturelle des massifs rocheux}

Les compressions internes d'un massif dues à la pesanteur et aux poussées orogéniques, sont évidemment un élément important caractérisant son état mécanique. La distribution locale des contraintes sera nécessairement irrégulière du fait des cassures, ou des différences de dureté des zones diverses du massif. On a constaté que les compressions internes n'étaient en rien liées à une direction donnée. Il semble même que se vérifie dans une certaine mesure la théorie de Heim dite de l'hydrostatisme des tensions naturelles. En général la compression naturelle minimum reste en tout point du massif relativement élevée. Il est évident que les conditions naturelles de compression assurent une stabilité de la roche qu'on peut considérer comme indéfinie.

\section{Compressibilité des roches}

Tout comme la résistance intrinsèque, la compressibilité des roches s'avère différente sur échantillons compacts et sur la masse clivée.

Sur échantillon compact, l'essai de compression fait apparaître même pour les zones les plus dures un raidissement léger de la roche lorsqu'une certaine compression est dépassée. C'est le serrage initial. A la décompression, il y a desserrage. Ce serrage et ce desserrage sont pratiquement réversibles, et on peut les attribuer à une fermeture élastique de petits espaces intergranulaires.

Lorsque la compression avoisine la rupture, il y a fléchissement de l'élasticité, sans doute par ouverture de fissures microscopiques préparant l'effondrement. Avec le temps, et en triple étreinte, on peut obtenir un fluage.

$\mathrm{Si}$ nous passons maintenant aux roches fissurées et décomprimées, et par exemple à celles du pourtour des souterrains, leur comportement est très différent de celui de la roche compacte (fig. 9). Les premières compressions y produisent aussi un serrage. Mais celui-ci, qui résulte de la fermeture du clivage, est important et irréversible. Ce n'est que lorsque la roche est serrée sous forte étreinte qu'elle peut approcher de l'élasticité des échantillons compacts. Le module d'élasticité alors atteint est le module vrai, équivalent du module « dynamique » mesurable par des méthodes d'auscultation sonore. Mais en gé-

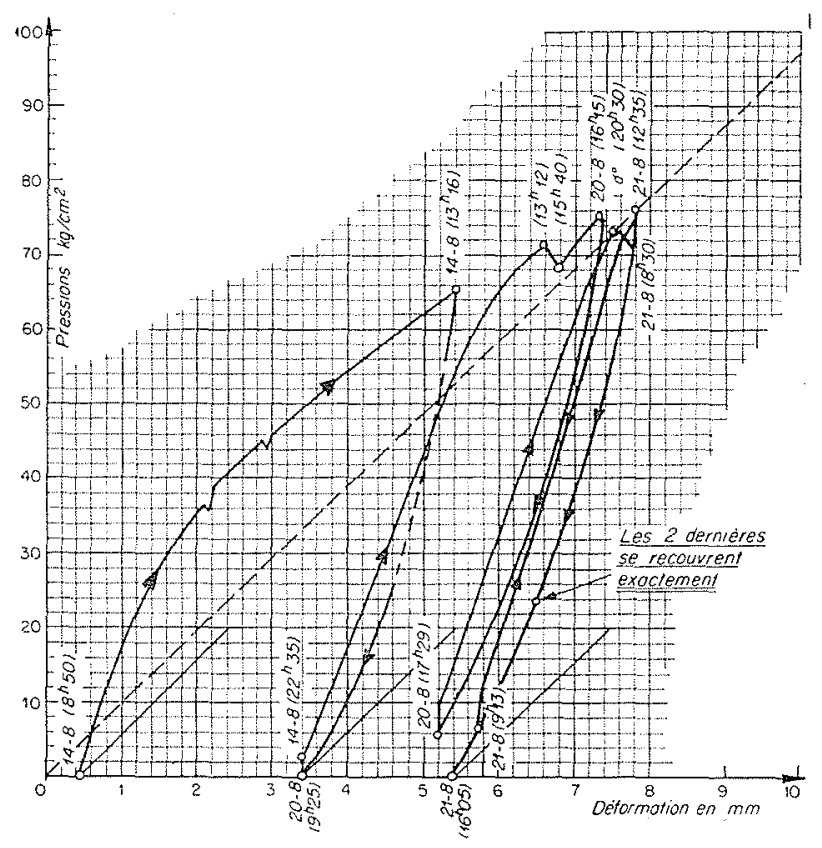

Fig. 9. - Compressibilté DU rocher a Malgovert.

néral, la roche fissurée a un comportement qui n'est ni élastique linéairement ni même réversible.

Lorsqu'elles dépassent les conditions limites de glissement, les fortes charges produisent d'ailleurs des déformations dont une partie est différée et qui donnent l'apparence de mouvements de fluage. Tel est le cas lorsqu'on s'approche des conditions du poinconnement.

\section{Les mesures sur les roches}

Les instruments de mesure sur les roches peuvent être divisés en deux grands groupes, ceux destinés aux mesures de compressibilité, ceux destinés à la détermination des pressions internes du rocher.

\section{$1^{\circ}$ Mesures de compressibilité.}

Les vérins permettent d'exercer sur une paroi de niche ou de galerie un effort considérable, pouvant être appliquée par cycles de charge ou de décharge d'une durée quelconque. Ils ont été 
fréquemment utilisés pour la détermination de la compressibilité du rocher. C'est là une pratique critiquable, car aux faibles charges, la compressibilité de la paroi décomprimée est exagérée et aux fortes charges, un poinçonnement se produit.

Par contre, l'essai au vérin peut donner la valeur de poinçonnement du rocher. Pour une galerie en charge, et même pour un barrage, un tel essai n'a toutefois qu'un faible rapport avee les conditions réelles d'utilisation du terrain. En outre, l'essai an vérin doit se faire nécessairement en niche ou en galerie étroite, et non sur: un plan indéfini comme le voudrait la théorie.

Les dilatomètres à poche de caoutchoue sont en somme des instruments reproduisant en réduction les cavernes d'essai dont nous reparlerons, et essayant de remplacer les vérins. Ils sont done passibles des critiques adressées à ceux-ci. Des difficultés constructives ont d'ailleurs empêché l'emploi étendu de ces appareils.

L'auscultation sonore utilise la mesure de la propagation de la vitesse du son aux parois des souterrains afin de déterminer le module d'élasticité moyen ou local de la roche en place. Les roches les plus dures et les plus résistantes ont un coefficient d'élasticité élevé. La fissuration des roches ralentit considérablement la propagation du son. L'auscultation sonore permet done d'affecter un coefficient de qualité à chaque section étudiée, coefficient qui correspond fidèlement aux indications que peut donner un examen géologique minutieux, comme on l'a vérifié sur la galerie de Pragnères. Il est possible que l'on puisse tirer, dans un proche avenir, de cette méthode d'auscultation, des résultats encore plus intéressants.

La mesure sur échantillon au laboratoire donne le module d'élasticité maximum de la roche et le serrage élastique de la roche.

\section{$2^{\circ}$ Détermination DES PRESSIONS INTERNES DU ROCHER.}

La plupart des méthodes de mesure des pressions internes des massifs rocheux utilisent le principe de la libération des contraintes. Il s'agit de faire disparaître, par une saignée, la compression dans la roche, et de relever les déformations qui résultent de cette saignée sur une base donnée.

L'application la plus ancienne de cette méthode comporte l'utilisation d'extensomètres mécaniques pour mesurer les déformations et la réalisation de la saignée au marteau piqueur (fig. 10).

L'emploi de jauges extensométriques à résistance dont l'encombrement est très faible, a permis la réalisation de la saignée par carottage de $250 \mathrm{~mm}$ de diametre. Les extensometres à rísistance peuvent être disposés en rosette. Ils donnent alors les tensions principales à la paroi étudiée. La difficulté est de trouver dans les souterrains des parois susceptibles de recevoir les jauges dans de bonnes conditions.

Le dispositif du professeur Bertmen utilisé à Roselend, est constitué par un extensomètro a 3 directions dont le corps cylindrique porte 6 palpeurs élastiques dont le mouvement déplace 6 billes apparentes sur la face visible du corps. L'extensomètre peut-être calé dans un forage de

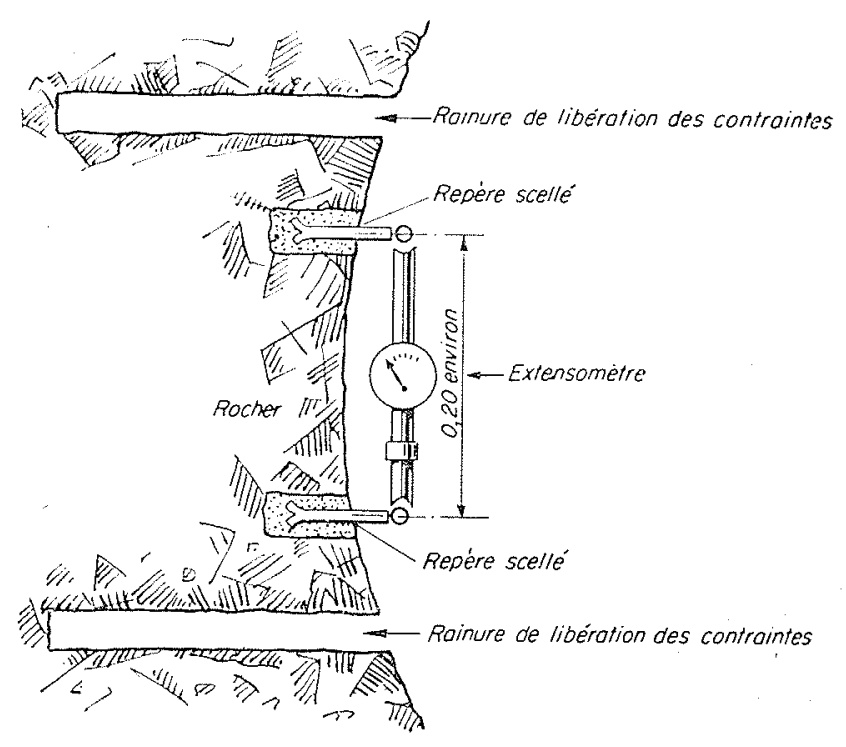

Fig. 10. - Dispositrf de mesure de compressions AUX PAROIS.

$56 \mathrm{~mm}$ de diamètre à une profondeur pouvant dépasser le mètre. Les billes du cadran de l'extensomètre sont photographiées avant carottage. Le rocher est ensuite décomprimé par un carottage de $250 \mathrm{~mm}$ environ et le cadran est photographié à nouveau. Les déplacements des billes sont étudiés par la comparaison des deux photographies. Des billes fixes servent à repérer la position vraie de chaque image. Ce dispositif a reçu d'ailleurs récemment d'importants perfectionnements, qui en facilitent beaucoup l'emploi sans en modifier le principe.

Toutes les méthodes ci-dessus opèrent par différence entre une position finale et une position initiale, et elles utilisent la décompression totale: Il s'ensuit que le coefficient de compressibilité de la roche est mal défini, en raison du desserrage en fin de décompression, lequel équivaut à un effondrement pour les roches fissurées. Aussi a-t-on mis en doute la valeur des résultats obtenus par ces méthodes et a-t-on proposé des procédés quelque peu différents. 
Afin d'éliminer l'incertitude pesant sur les coefficients d'élasticité, on a songé à ramener le rocher à la position initiale à l'aide d'une pres. sion mesurée. Pour cela, la décompression dans l'étal intermédiaire est réalisée à l'aide d'une saignée plane aménagée en vue de l'inclusion ultérieure d'un vérin plat Freyssinet (fig. 11). Les déformations du rocher sont vérifiées par une

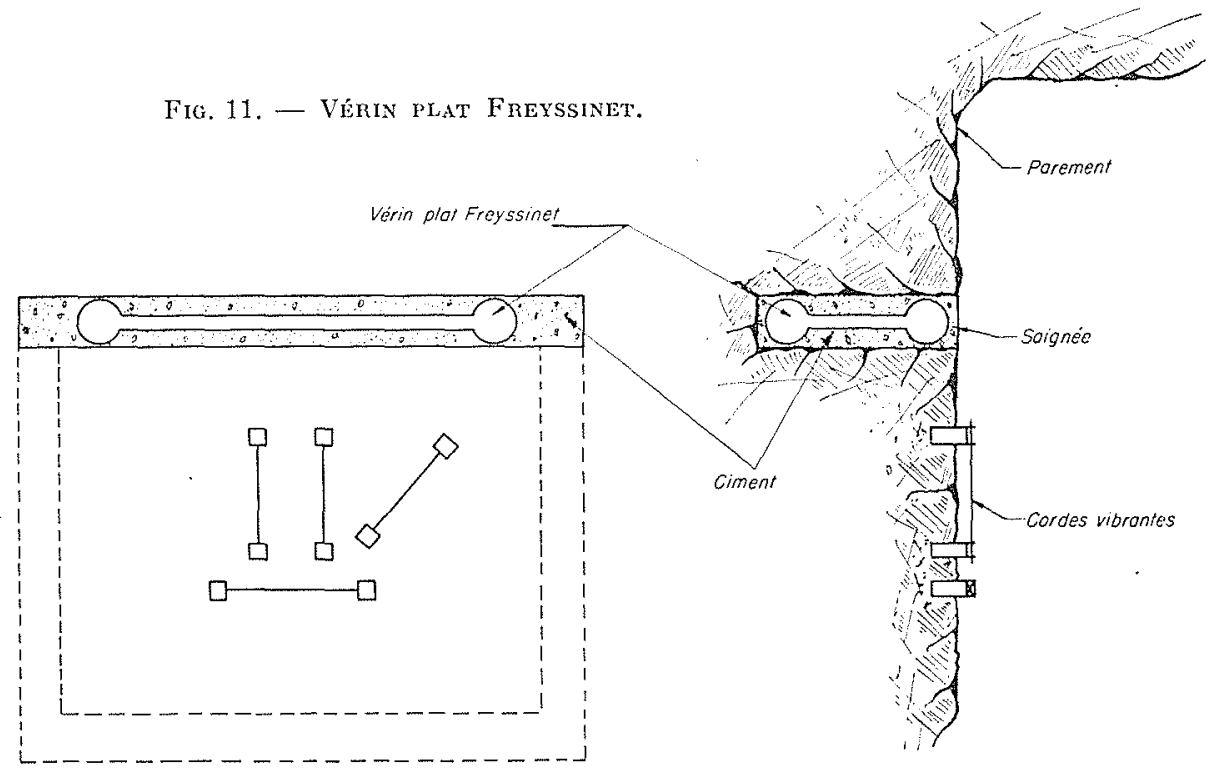

série de cordes vibrantes nues convenablement disposées. Le vérin plat étant cimenté dans la fente, une pression progressive lui est appliquée afin de ramener les cordes vibrantes à leur tonalité initiale.

Un autre perfectionnement consiste à suivre de façon continue l'allure de la déformation en fonction de la profondeur de carottage, et par suite de la décompression. Nous avons fait construire un appareil à 3 directions où les déformations sont transmises électriquement par variations d'inductance, à l'aide d'un câble qui peut traverser la tige de la sondeuse. Le décarottage est effectué dans le diamètre de $235 \mathrm{~mm}$. La profondeur que peut atteindre l'appareil est prévue de l'ordre de plusieurs mètres, suffisante pour étudier la répartition des pressions au pourtour des galeries. Le fait que l'on peut suivre la déformation point par point permet, non seulement de vérifier que l'allure de la courbe de déformation est normale, mais en outre, de choisir n'importe quelle partie de la courbe pour la transformation des déformations en contraintes. Le tarage doit être alors fait d'après un modèle réduit de forage effectué sur une matière parfaitement élastique, la forme du forage ne se prêtant pas àu calcul. La méthode exige l'utilisation d'un module d'élasticité; il suffira do choisir une phase favorable de la décompression, par exemple celle du début, pour que l'application du module dynamique soit justifiée.

Pour les mesures à la paroi, le même principe. peut être utilisé. C'est ce qui a été fait dans la

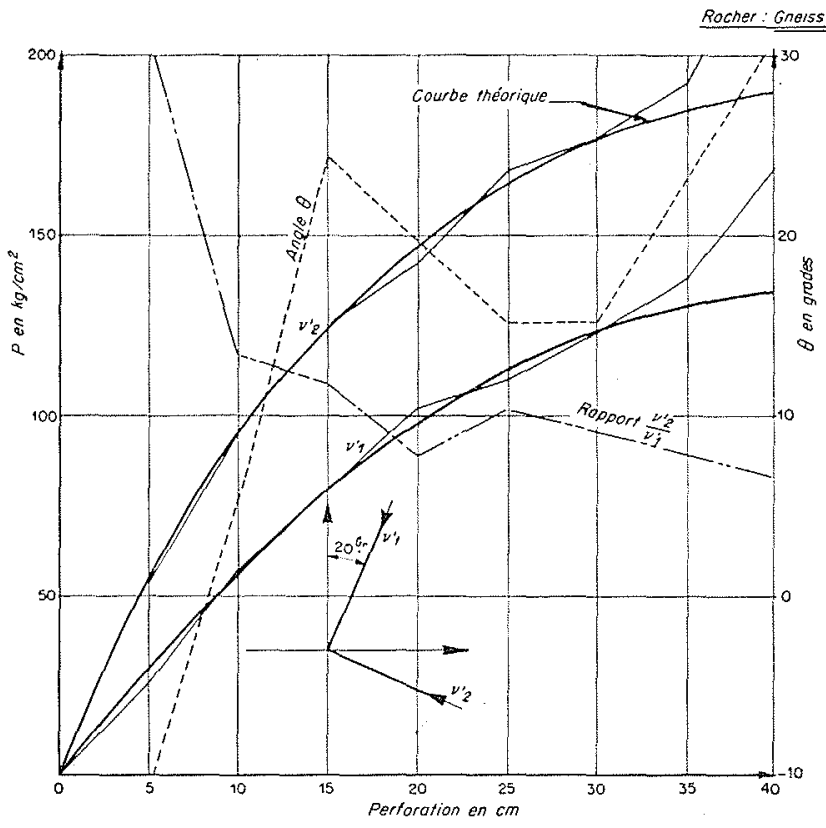

Fig. 12. - Chute de Montpezat. Contraintes principales préexistantes Mesures à l'extensometre l'Hermite (erreur $上 5 \mu$ ). 
souterrain de la conduite blindée de Montpezat (fig. 12).

Les mesures à la paroi doivent d'ailleurs être conjuguées avec les mesures en profondeur. Prises isolément elles fournissent à tout le moins un bon indice de la résistance de la roche à la compression. Cet indice est d'autant plus précieux qu'il n'existe pas d'autre méthode d'apprécier la résistance intrinsèque du rocher, si ce n'est l'essai au vérin. Du fait que la paroi supporte les contraintes mesurées, on est assuré de sa stabilité sous ces contraintes.

\section{$3^{\circ}$ Etanchéité.}

II reste enfin à mesurer sur place l'étanchéité de la roche. Cette mesure est faite souvent à l'aide de forages de $46 \mathrm{~mm}$, profonds d'une dizaine de mètres et obturés par des bouchons à une profondeur donnée (fig. 13).

On constate que les fuites croissent avec les pressions, d'abord lentement, en accord avec la mise en compression élastique du rocher, puis beaucoup plus rapidement à partir d'une pression critique qui correspond au dépassement de

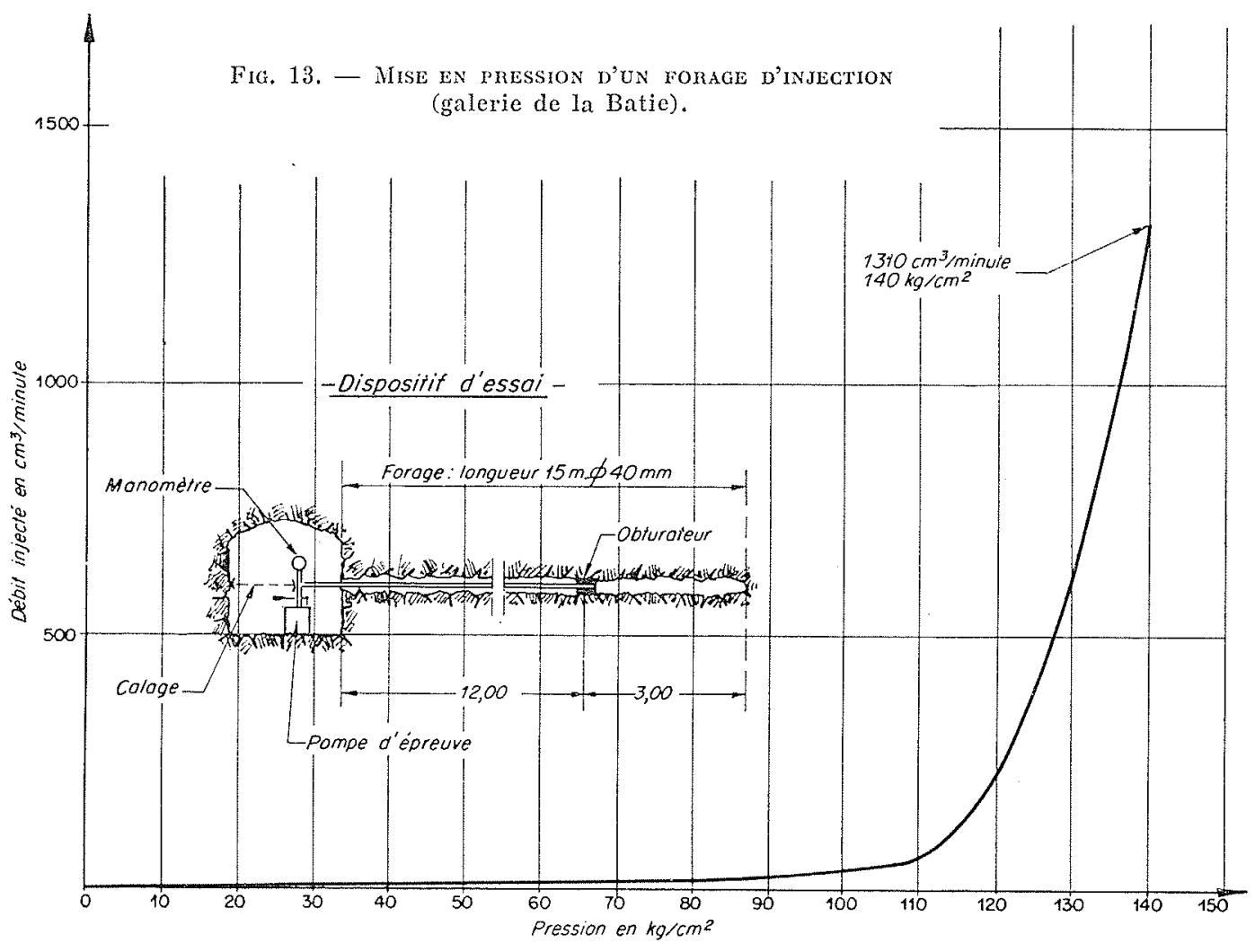

la compression naturelle minimum du massif et a l'ouverture des fissures. L'enregistrement des fuites en fonction de la pression donne donc un moyen de connaître la pression intérieure du rocher.

\section{Dispositifs d'essais en caverne}

Les mesures isolées peuvent être complétées par des essais effectués sur des tronçons de souterrains mis en pression, dont on mesure les pertes et les dilatations diamétrales. Un tel essai a, par exemple, été entrepris à Bort sur une ca- verne revêtue, véritable modèle à girande échelle de l'ouvrage réel (figg. 14).

De tels dispositif's donnent des résultats globaux qui permettent de vérifier la justesse du dessin adopté pour un revêtement, mais sont souvent d'une interprétation très complexe.

\section{Aciers et bétons}

Les bétons et les aciers sont suffisamment connus pour qu'il soit inutile de les étudier ici. Signalons toutefois que le béton est sujet comme les roches à serrage ou à fluage et en plus qu'il 

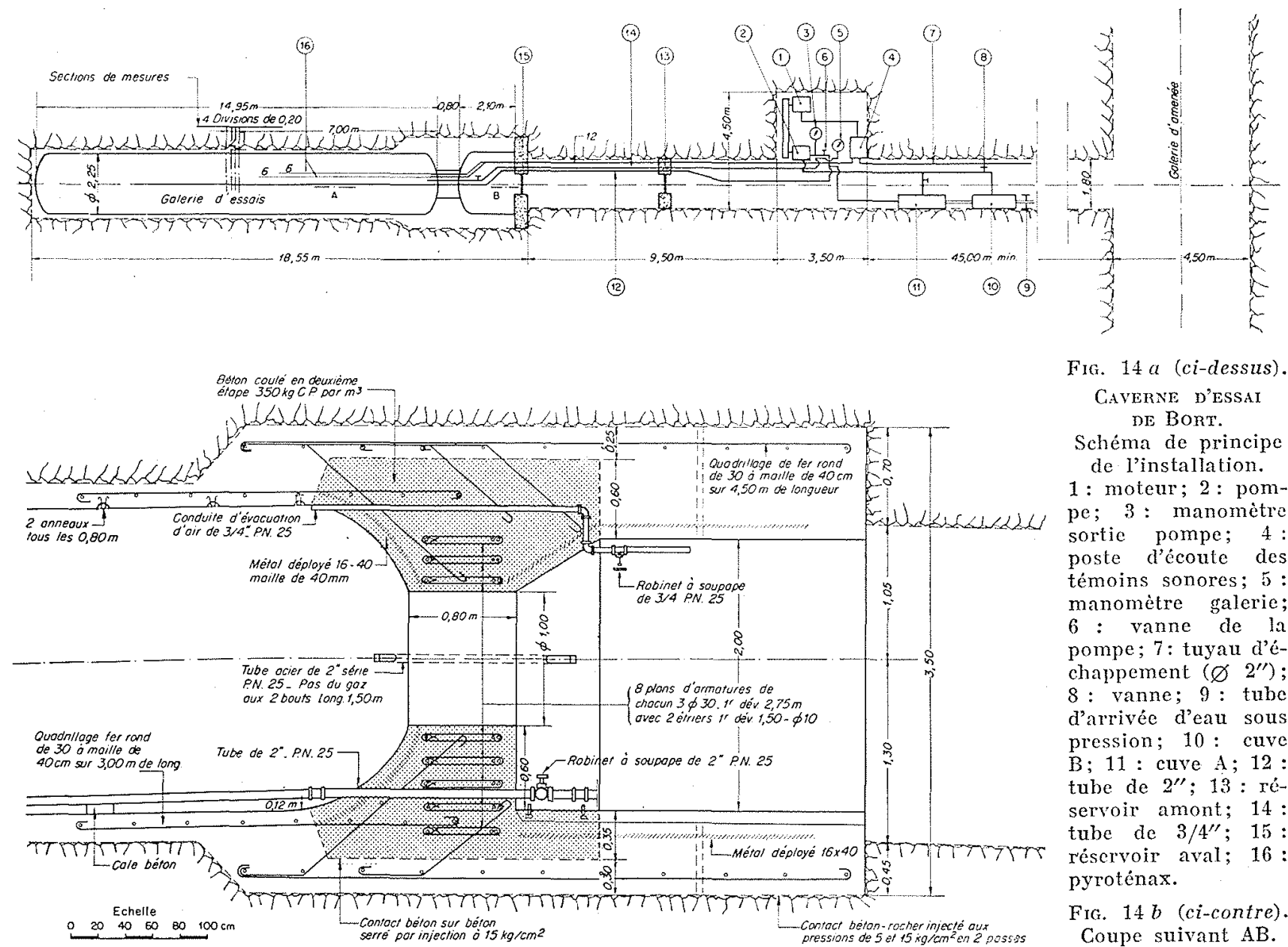

Fig. $14 a$ (ci-dessus).

Caverne d'essai DE BORT.

Schéma de principe de l'installation. 1: moteur; 2 : pompe; 3 : manomètre sortic pompe; 4 : poste d'écoute des témoins sonores; 5 : manomètre salerie; 6 : vanne de la pompe; 7: tuyau d'échappement $\left(\varnothing \quad 2^{\prime \prime}\right)$; 8: vanne; 9 : tube d'arrivée d'eau sous pression; 10 : cuve B; 11 : cuve A; 12 : tube de $2^{\prime \prime} ; 13$ : réservoir amont; 14 : tube de $3 / 4^{\prime \prime} ; 15$ : réservoir aval; 16 : pyroténax.

FIG. $14 b$ (ci-contre). Coupe suivant $\mathrm{AB}$.

peut être affecté par le retrait. Il peut enfin être attaqué par les eaux très pures ou alcalines. Il importe donc qu'il soit soustrait à la circulation des eaux souterraines.

L'acier est lui aussi sujet à fluage. En ce qui concerne les blindages, les déformations permanentes devraient être au maximum évitées dans le métal. Toutefois il n'a jamais été démontré que ces déformations, ou la mise en tension de très longue durée, puissent diminuer la résistance à la rupture, comme c'est le cas à chand.

\section{Conditions de réalisation des conduites forcées souterraines}

\section{L'Excavation.}

Passons à la réalisation des conduites forcées souterraines. C'est évidemment par l'étude du rocher que doit débuter tout projet. Malheureu- sement, il n'existe encore aucun moyen de connaître parfaitement le sous-sol avant excavation. D'autre part les reconnaissances sont limitées et c'est au géologue que seront demandés les. renseignements qui font défaut. La position de la conduite dans le massif qui doit la contenir devra réserver une couverture rocheuse suffisante, qui doit en tout point si possible dépasser largement 40 à $50 \%$ de la pression de marche mesurée en mètres d'eau. La pente de la conduite sera fixée en fonction de la topographie et surtout du mode d'excavation prévu. Si le souterrain doit être attaqué de bas en haut, la pente de $40^{\circ}$ environ, ou pour préciser, dont la tangente est comprise entre 85 et $100 \%$, permet le glissement naturel des déblais vers les recettes au bas des galeries. Mais pour un marinage par wagonnets sur monorail, comme à Montpezat, la pente de $47 \%$ séra suffisante. Les souterrains ont été attaqués parfois par le haut. Dans ce cas, le puits de chute vertical a été adopté fréquemment.

Le diamètre de la conduite sera déterminé par 
un calcul économique mettant en balance pertes d'énergie et charges résultant des investissements. Le diamètre devra donc diminuer, de préférence par paliers, avec la pression. Un gros bénéfice sera souvent obtenu par la possibilité de réunir deux conduites en une seule, ce qui donnera des pertes de charge plus faibles, et des coups de bélier moins importants.

L'excavation sera poursuivie avec le maximum de précautions. Il faut signaler le soin avec lequel de telles excavations sont conduites en Norvège. Le contour de l'attaque est délimité par

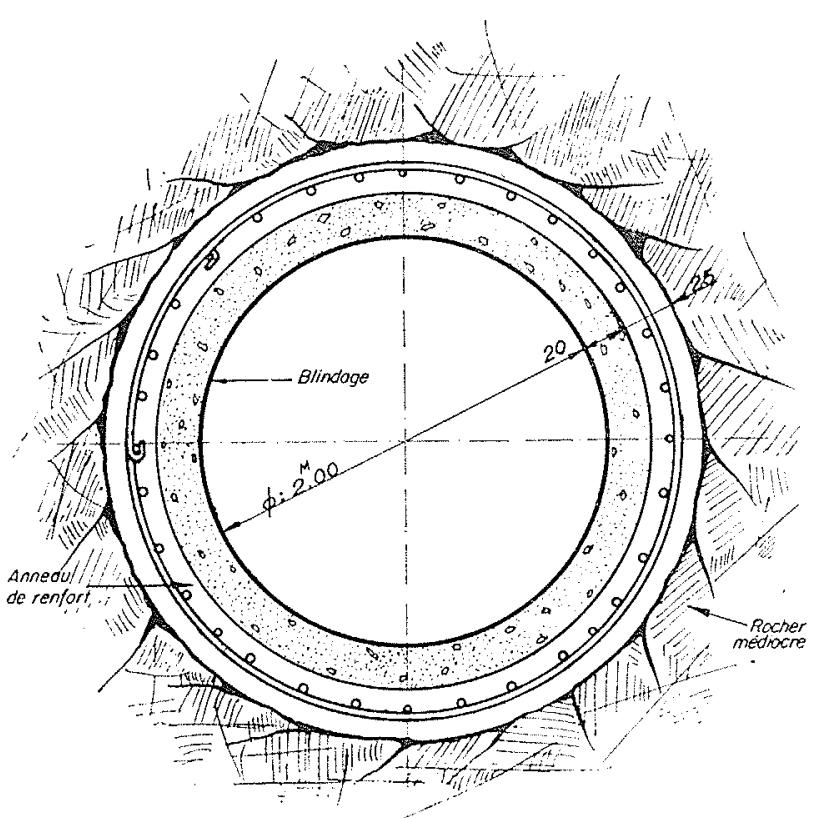

Fig. 15. - RENFORCEMENT EN BÉTON ARMÉ povn PUTS DE CHUTE.

des forages serrés, chargés modérément, afin d'obtenir, même dans le granit diaclasé, une surface régulière et peu ébranlée. Quelques expériences, malheureusement isolées, ont montré que les tirs pouvaient faire se déplacer les parois d'un souterrain de plus d'un centimètre vers l'intérieur. Cela correspond à une décompression considérable qu'il sera évidemment cô̂teux de rattraper par la suite.

\section{Revêtements}

En plus des précautions de tir, il serait utile de ne point laisser le rocher nu et sans soutènement durant de longs mois. Pour les ouvrages importants, il semble que l'on puisse conseiller un prérevêtement de béton ordinaire, suivant du plus près possible le front d'attaque, et assurant ainsi protection et soutènement. Ce prérevêtement est encore plus nécessaire si la roche est fissurée et attaquable à la longue par l'air et par l'humidité.

Si la galerie rencontre une zone broyée ou faillée, la meilleure méthode sera de constituer au pourtour de l'excavation un anneau de béton armé assez épais, dans lequel on fera travailler les armatures à un taux élevé (fig. 15). Il ne s'agit plus ici, en effet, d'assurer l'étanchéité, et les critiques que nous avons faites contre les revêtements armés qui se fissurent n'ont plus à être retenues. Nous verrons qu'un tel anneau, du fait de son énorme inertie, est bien préférable à un épaississement des blindages.

\section{Choix du revêtement}

La hauteur de chute, la nature du rocher et Ies résultats des mesures sur le terrain donneront les éléments du choix de la section. En général, le rocher ne sera ni parfaitement inaltérable, ni rigoureusement étanche, et il devra être revêtu. Aucun puits de chute non revêtu ne dépasse d'ailleurs à ce jour la pression statique de 165 mètres.

Pour une chute inférieure à $150 \mathrm{~m}$, le béton armé pourra être envisagé. Jusqu'à $250 \mathrm{~m}$ de pression, le béton précontraint peut être adopté sans chemisage. Pour les pressions plus fortes, l'étanchéité exigera nécessairement un blindage.

Lorsqu'un blindage sera nécessaire, il faudra qu'il puisse résister aux éventuelles corrosions, aux flexions durant le transport et le montage, et enfin aux sous-pressions. C'est dire que l'épaisseur du blindage sera le plus souvent à peu près imposée. Nous répétons ici qu'il ne faut songer à prévoir des galeries de drainage qu'en cas de nécessité absolue, et qu'on évitera en général tout ce qui pourrait éloigner la section transversale de la conduite, de la forme circulaire idéale. Mais bien sûr des rideaux d'injection et des drains, superficiels ou profonds, pourront être prévus pour améliorer l'assainissement du massif.

\section{Calcul des conduites}

Le calcul des conduites souterraines fait joner les élasticités différentes des anneaux d'acier, de béton et de rocher pour en tirer une proportion vraisemblable de répartition des efforts. Le coefficient d'élasticité du rocher est donc une donnée à laquelle on attache une grande importance. 
On oublie toutefois souvent que le rocher n'a pas plus que le béton un coefficient d'élasticité défini. Un rocher décomprimé par la perforation peut sous charge de longue durée, avoir un module anormalement bas. Le même rocher, bien serré par injections, peut au contraire retrouver à la fois l'état d'étreinte naturel indéfiniment stable et un module très élevé et à peu près égal au module dynamique (fig. 16). D'autant plus que le remplissage des cassures par le coulis de ciment peut le transformer en une véritable maçonnerie. Il est donc indispen-
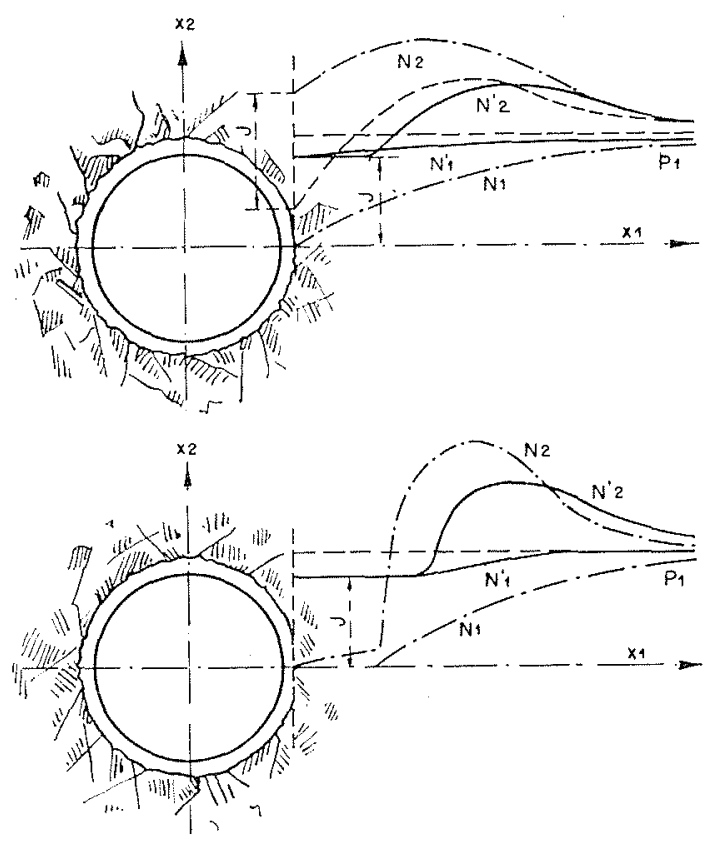

Fig. 16. - EFForts Après REVÊTEMENT ET INJECTIONS DE CMENT.

sable de serrer le rocher. Quelques mesures d'élasticité souvent limitées à des échantillons de roche saine, ne sauraient en dispenser.

Mais l'élasticité du rocher n'est pas tout. Encore faut-il que la résistance du massif soit suffisante. La règle du minimum de couverture donne une indication utile. Les mesures de compression naturelle précisent cette règle. Un rocher très comprimé est nécessairement peu compressible. Il est insensible aux diminutions de compression qu'amènera la mise en charge. Un souterrain peut supporter théoriquement une pression d'eau égale à la compression minimum relevée sur ses parois. Certains calculs pubilés dans la littérature technique font état d'une couronne de rocher fissuré par traction au pourtour des puits de chute. A notre avis, cela n'est pas admissible. Aucune traction ne peut et ne doit être tolérée dans la roche derrière le blindage. Si elle existait, il faudrait la supprimer par injection pour assurer la stabilité qui est ici une nécessité absolue.

Pour tenir compte du retrait thermique, on ménagera une marge d'environ $25 \mathrm{~kg} / \mathrm{cm}^{2}$ de compression supplémentaire dans le rocher et le béton et de près de $3 \mathrm{~kg} / \mathrm{mm}^{2}$ de traction dans la tôle ou les aciers du revêtement.

Si le terrain est meuble, par exception, la sécurité ne pourra être assurée contre les fuites accidentelles que si la condition de renard est respectée, ce qui demandera une hauteur de couverture voisine de la charge de service.

Les calculs supposent aussi que le blindage ne va subir que des tractions simples, à l'exclusion de toute flexion. C'est là une hypothèse bien imprudente, car la roche est loin d'être isotrope. Aussi le blocage en béton au pourtour de la conduite doit-il avoir une forme et une composition régulières, et une épaisseur suffisante pour amortir l'anisotropie du terrain. Au besoin, le béton pourra être armé. Ce n'est que dans quelques cas favorables que l'on pourra sans crainte diminuer au minimum l'espace entre l'acier et le terrain.

\section{Serrage du rocher}

Le supplément de dépenses nécessité par le serrage du rocher sera compensé largement par la sécurité obtenue. A l'heure actuelle, le serrage par injection au pourtour des viroles dans un espace annulaire a donné lieu à des réalisations pratiques très intéressantes. Faute de mieux, et au minimum, on prévoiera des injections très poussées de remplissage et de serrage aux contacts béton-rocher et acier-béton.

\section{RACCORDEMENT A L'USINE.}

Logiquement, une usine souterraine devrait accompagner toute conduite souterraine. Il n'en a pas toujours été ainsi, pour des raisons diverses.

Mais cette disposition empêche le plus souvent d'enfoncer la conduite dans le terrain, ce qui compromet la participation du rocher à sa résistance. En outre, le passage du souterrain profond à la surface constitue toujours un point d'articulation délicat. Ce passage est fréquemment affecté de fissures transversales désolidarisant les zones profondes des zones extérieures, et créant les joints de dilatation qu'on n'a pas pu prévoir. On peut signaler de nombreux accidents à ce passage dans des galeries blindées ou non, notamment à Saint-Geniez-O-Merle, à Lardit, à Marèges et à l'étranger. L'adoption d'une 
forte couverture rocheuse est donc une mesure prudente, tant pour l'usine que pour la conduite, même si la forte couverture entraîne des accès un peu plus coûteux. La zone superficielle, toujours très affectée par les intempéries, peut avoir jusqu'à $40 \mathrm{~m}$ d'épaisseur.

\section{Protection par vannes.}

Une conduite forcée souterraine bien exécutée doit procurer une sécurité presque totale. La question pourrait se poser de savoir si on doit prévoir en tête d'une telle conduite les organes de fermeture habituels pour les conduites aériennes. Si la conduite est bloquée dans le rocher sans drainage, une fissuration ne constituera qu'un incident d'exploitation, sans conséquences graves. La situation est toute différente si la conduite comporte un drainage ou si l'éclatement est proche des vannes d'arrêt au collecteur ou de la surface du sol, et si l'eau peut s'échapper en trombe à la suite de crevaison. Les dégâts alors seront considérables et la fer-

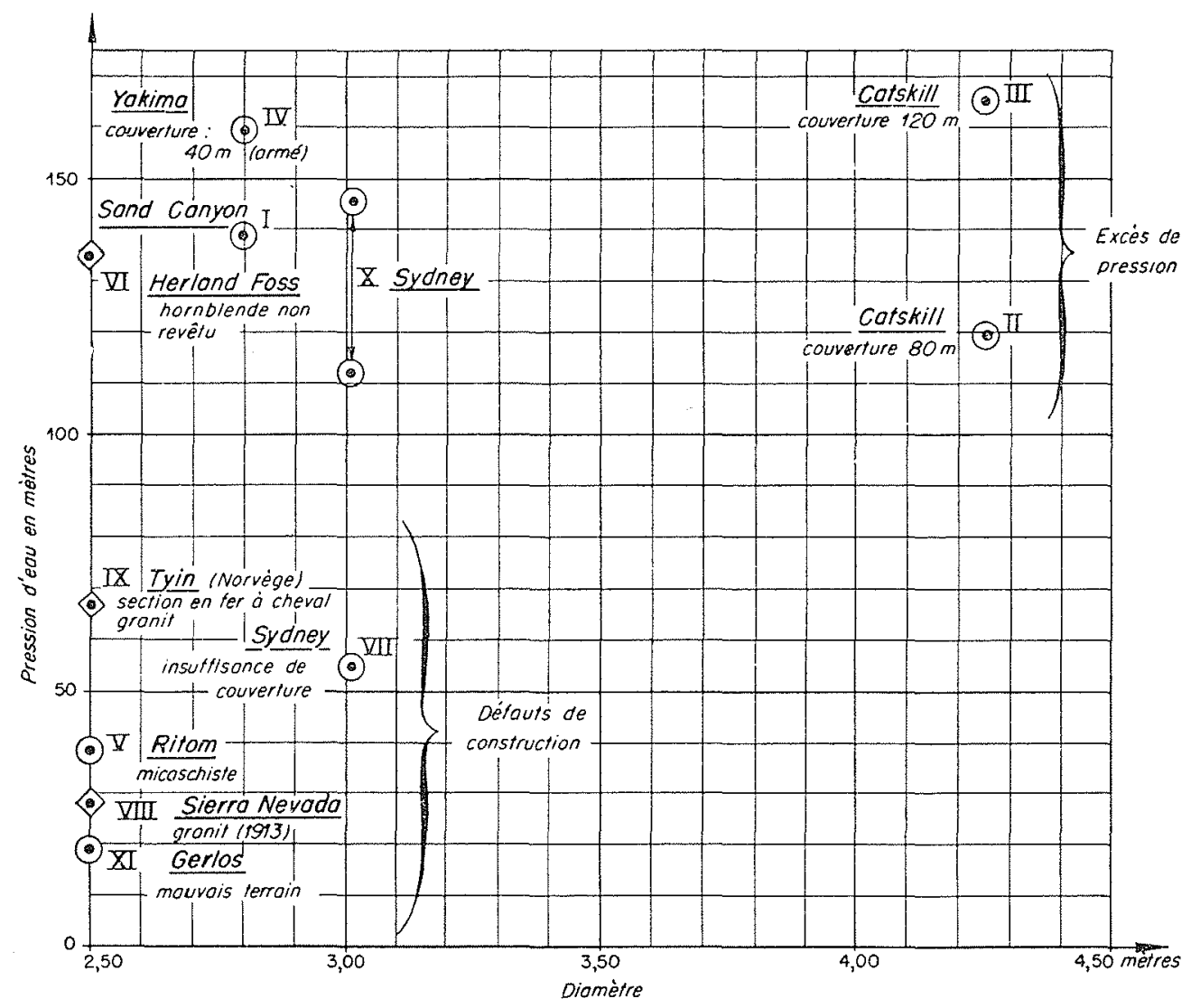

Fig. 17. - Ruptures de Galeries en charge (fissurations longitudinales avec fuites).

meture des vamnes ne pourra d'ailleurs qu'en diminuer l'ampleur. La dépense de vannes constitue une assurance dont on devra peser les charges et les avantages. Il semble que l'isolement des vannes de pied dans une galerie séparée, ou même dans des chambres isolées, ou que le renforcement des collecteurs, soient plus efficaces que la protection de la conduite par des vannes de tête. Mais une doctrine reste encore à établir.

\section{Collecteur.}

Les collecteurs sont des ouvrages de réalisation coûteuse, et la partition des débits des conduites souterraines pose parfois des problèmes difficiles. Heureusement, la technique de la soudure a fait ces dernières années des progrès étonnants, notamment aux Etats-Unis, puisqu'on y réalise la jonction d'éléments en acier spécial de $100 \mathrm{~mm}$ d'épaisseur travaillant à des taux de 
traction très élevés. Les pressions de service de certains réservoirs pour l'industrie chimique ont atteint $400 \mathrm{~kg} / \mathrm{cm}^{2}$. Les collecteurs de conduites forcées ne peuvent que profiter dans la période à venir des progrès déjà accomplis dans le domaine de la soudure et de son contrôle.

\section{L'expérience recueillie sur les puits de chute}

C'est l'expérience gagnée en cours d'exploitation des puits de chute qui peut seule en justifier pleinement les dispositions (fig. 17). A ce point de vue, les accidents ont été et seront toujours de sévères mais d'utiles leçons pour l'ingénieur. Il faut remercier tous ceux qui ont aidé à faire connaître ces accidents et à en dégager les enseignements. Le plus souvent, leur étude a fait apparaître quelque aspect nouveau des problèmes par lequel avaient été rendues inutiles les précautions du constructeur.

En ce qui concerne les ouvrages bruts en rocher, nous savons qu'il est difficile de dépasser la pression de $150 \mathrm{~m}$. Le puits de Svelgen creusé en 1920 a certes tenu jusqu'à ce jour, mais non sans quelques fuites. A la même époque, deux autres puits avaient été creusés pour deux autres usines norvégiennes. L'un d'eux a dû être abandonné à la suite d'une crevaison et l'autre a dû être étanché à l'aide de garnissages et d'enduits locaux.

Les revêtements simples ne permettent pas d'améliorer considérablement la situation et leurs fissurations forment une liste très instructive.

Les revêtements armés eux-mêmes ont été affectés fréquemment de fissures qui leur ont fait perdre leur étanchéité. Nous avons déjà indiqué comment cet inconvénient pouvait être éliminé par la précontrainte qui est sûre si elle est bien conduite.

Nous ne reprendrons pas la liste des accidents dus aux mouvements longitudinaux des galeries blindées au voisinage des sorties de souterrains. L'exemple de cassure transversale que nous donnons ici pour un revêtement de béton est récent. Il s'est d'ailleur's accompagné de ruptures de soudures entre viroles dans une partie blindée et il avait été précédé d'incidents identiques à des dates antérieures. On peut noter que les accidents se répètent au cours des temps au voisinage des mêmes points sur les mêmes ouvrages.

Les déchirures longitudinales de blindages, par ouverture des viroles suivant une génératrice, ne sont pas des plus rares. La fissure ne coïncide qu'exceptionnellement avec une ligne de soudure. Par contre, elle suit presque toujours le bord d'une défectuosité du blocage en béton ou du rocher.

Ce fut le cas à Mese lors du premier éclatement à la mise en eau. Bien que le chemisage Marinonr soit très souple, la compressibilité excessive d'une zone broyée sur l'une des parois a provoqué la déformation dissymétrique de la tôle et sa déchirure.

C'est également au droit d'une faille que s'est produite la déchirure dont nous donnons la photographie. Cette faille avait été amollie et débourrée par des fuites. Le blindage de $6 \mathrm{~mm}$ d'épaisseur, était cependant suffisant pour supporter toute la pression sous une tension inférieure à la limite d'élasticité, en supposant la virole non appuyée. L'éclatement ne peut, à notre avis, s'expliquer que par des dissymétries et des moments fléchissants dans la tôle. Le même blindage avait été affecté de ruptures locales au cours de ses années antérieures de service, alors qu'il fonctionnait sous une pression plus réduite. L'éclatement a d'ailleurs nettoyé la faille jusqu'au sol, provoqué un jaillissement cxtérieur et laissé un cratère.

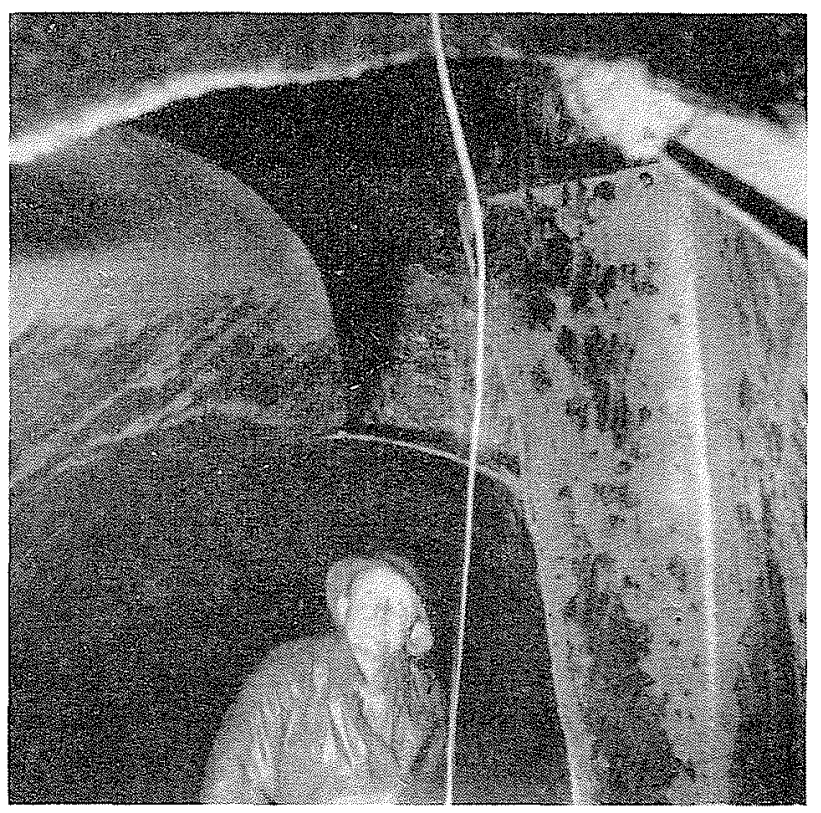

Fig. 18. - DËchinURE DE Btindage. 


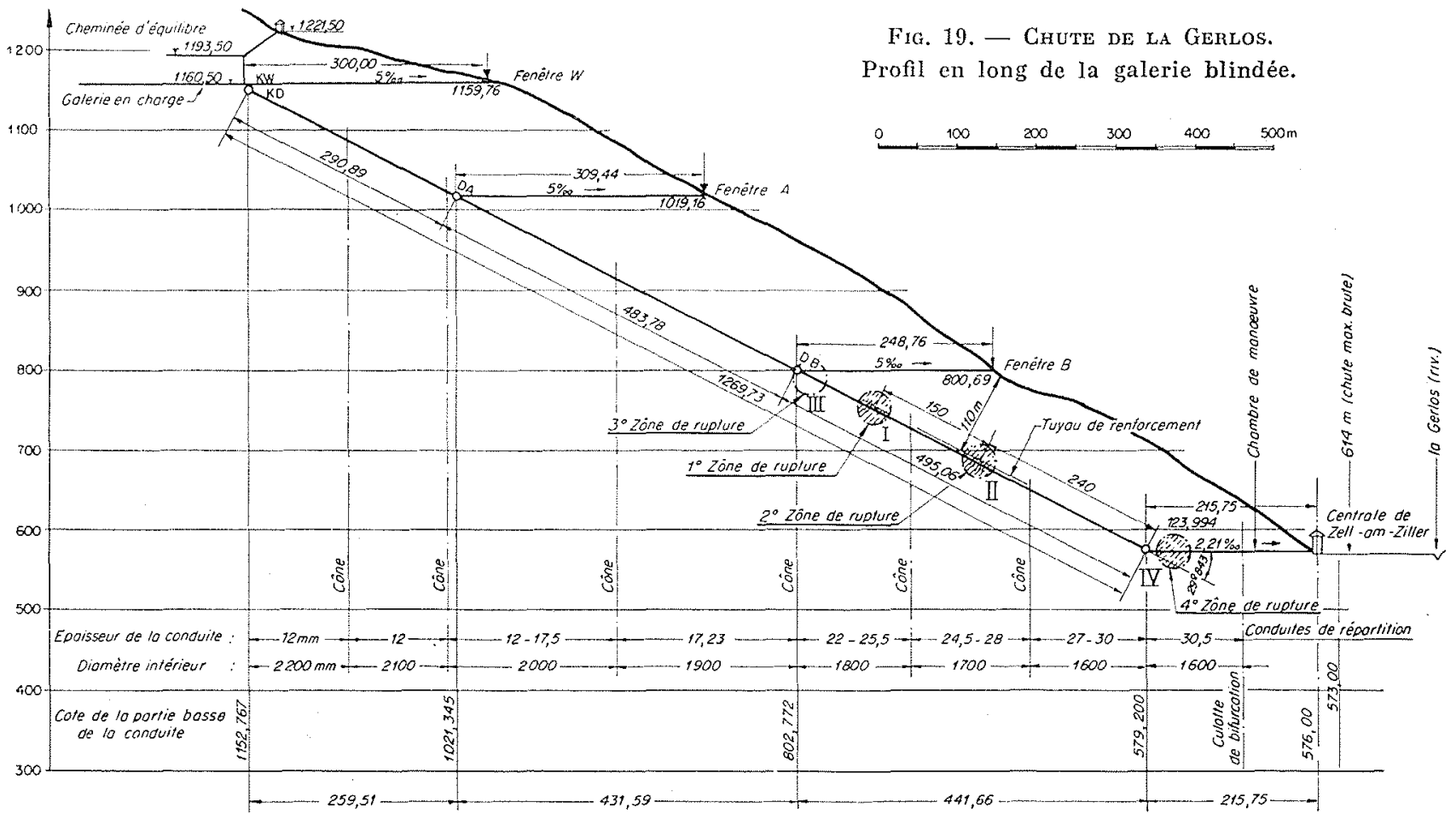

doprès "Tiroler Wosserkrottwerke" - Innisbruck

Grâce aux publications très précises des spécialistes autrichiens, qu'il faut féliciter de l'aide qu'ils apportent ainsi à l'art de l'ingénieur, les éclatements de la conduite forcée de la Gerlos peuvent jeter une certaine lumière sur les conditions de rupture d'un puits blindé (fig. 18). Les terrains traversés par la galerie d'amenée et par la conduite de la Gerlos sont médiocres, en majorité schisteux, et les couvertures sont faibles. Aussi doit-on signaler sur cette chute cinq accidents successifs de fissuration ou d'éclatement. Quatre de ceux-ci ont affecté la conduite forcée souterraine. Tous sont localisés dans le tiers inférieur de l'ouvrage, dont la pression est la plus forte et la couverture la pius faible. Liun d'eux est une rupture de soudure transversale due au retrait. Deux autres se sont produits à quelques secondes d'intervalle le 30 octobre 1945. On peut supposer que l'éclatement a débuté en 1 (fig. 19), et que la démolition du béton de blocage a provoqué la rupture 2 (fig. 20). On décida alors de combler partiellement la galerie de drainage. Après réparation et remise en eau, un éclatement se produisait le 29 septembre 1948 en 4 (fig. 21). Les dégâts causés à l'usine furent évidemment des plus graves, la galerie de drainage ayant conduit le flot directement dans la salle des machines. Le rocher et le béton autour des points de rupture furent profondément creusés par les eaux, soit à la rupture 1, soit à la

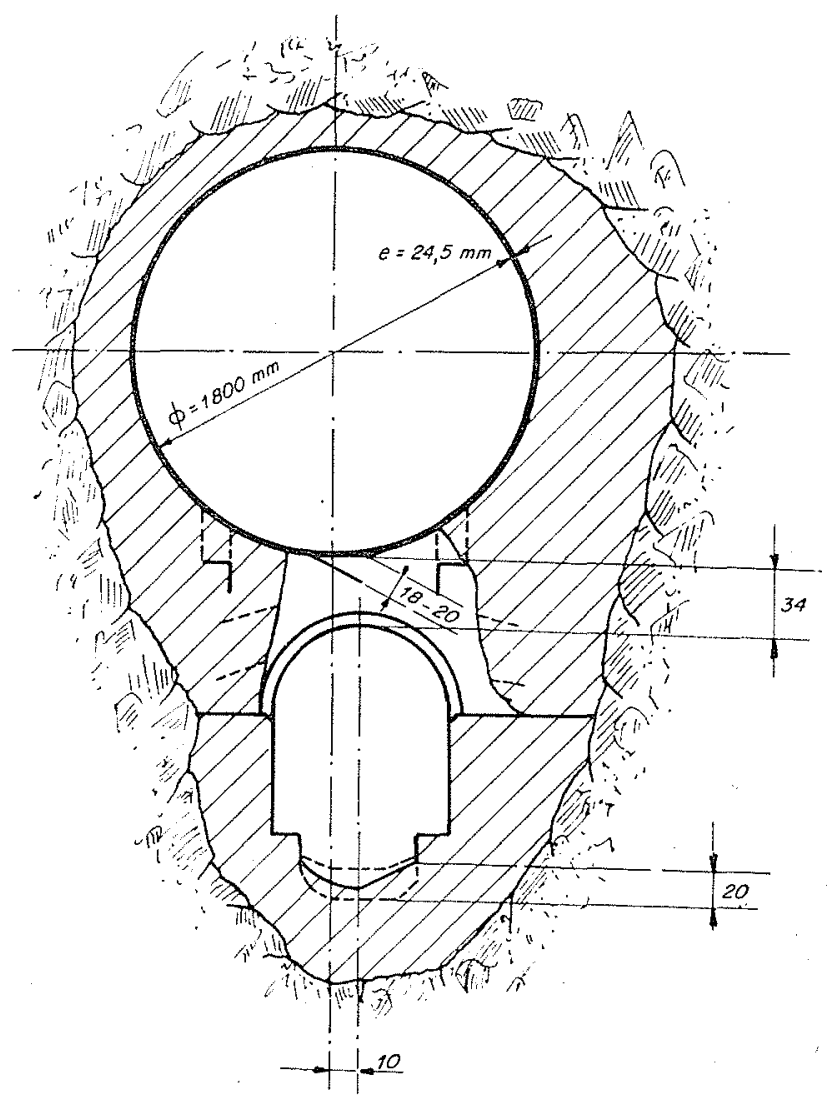

Fig. 20. - Puits de chute de la Gerlos. Rupture 1. 


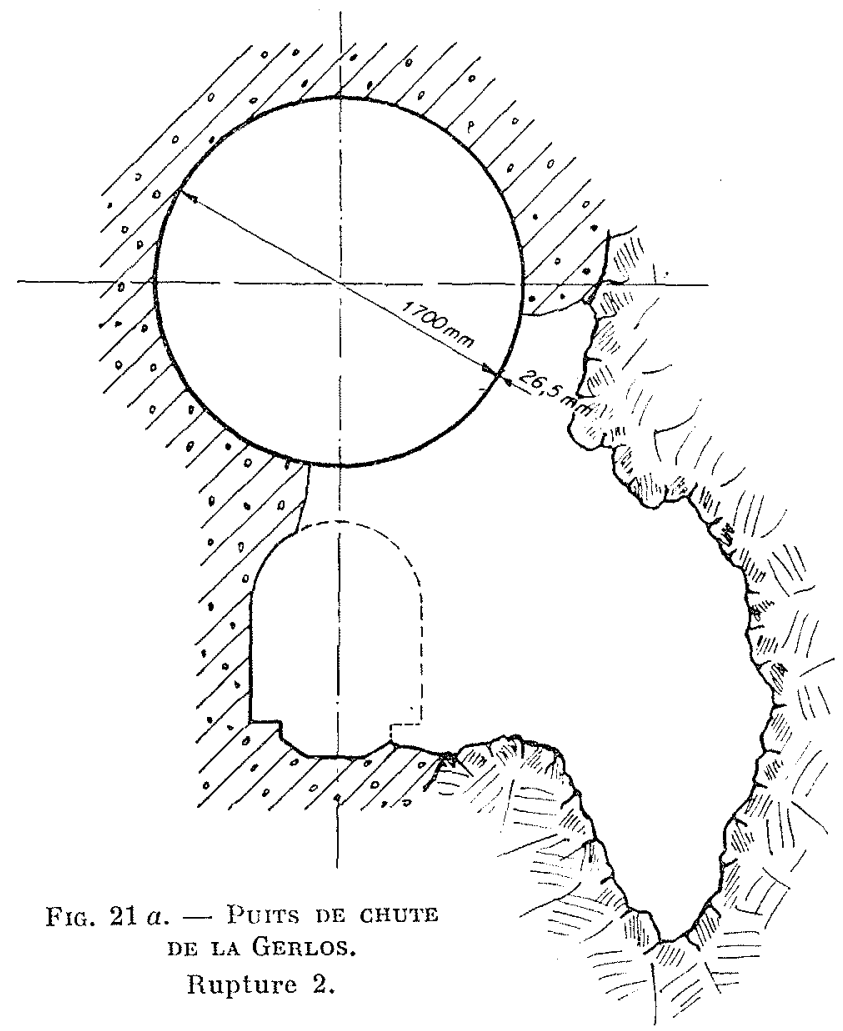

rupture 2, soit à la rupture 4. Il fut d'abord supposé que les ruptures provenaient de la qualité défectueuse du métal, lequel avait été livré pendant la guerre. Cependant, il faut noter que les éclatements se sont tous localisés au droit du vide de la galerie de drainage et qu'ils présentent un aspect trop homogène pour être dus

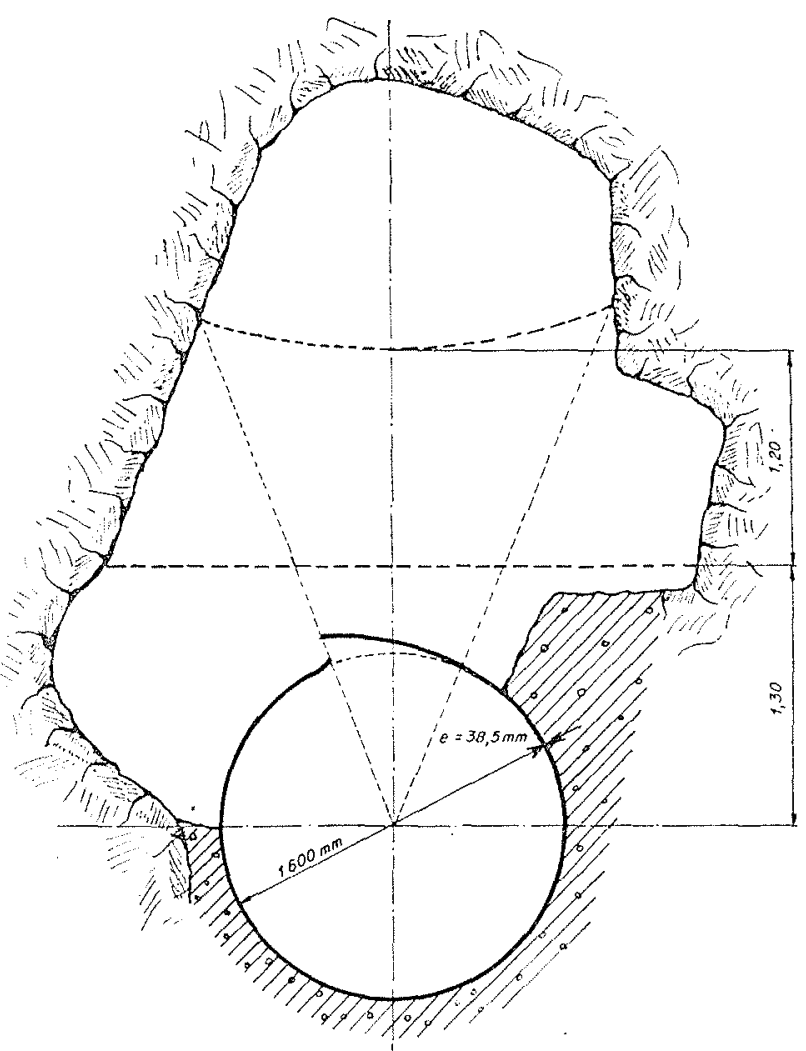

Fia. $21 b$. - Puits de chute de la Gerlos. Rupture 4.

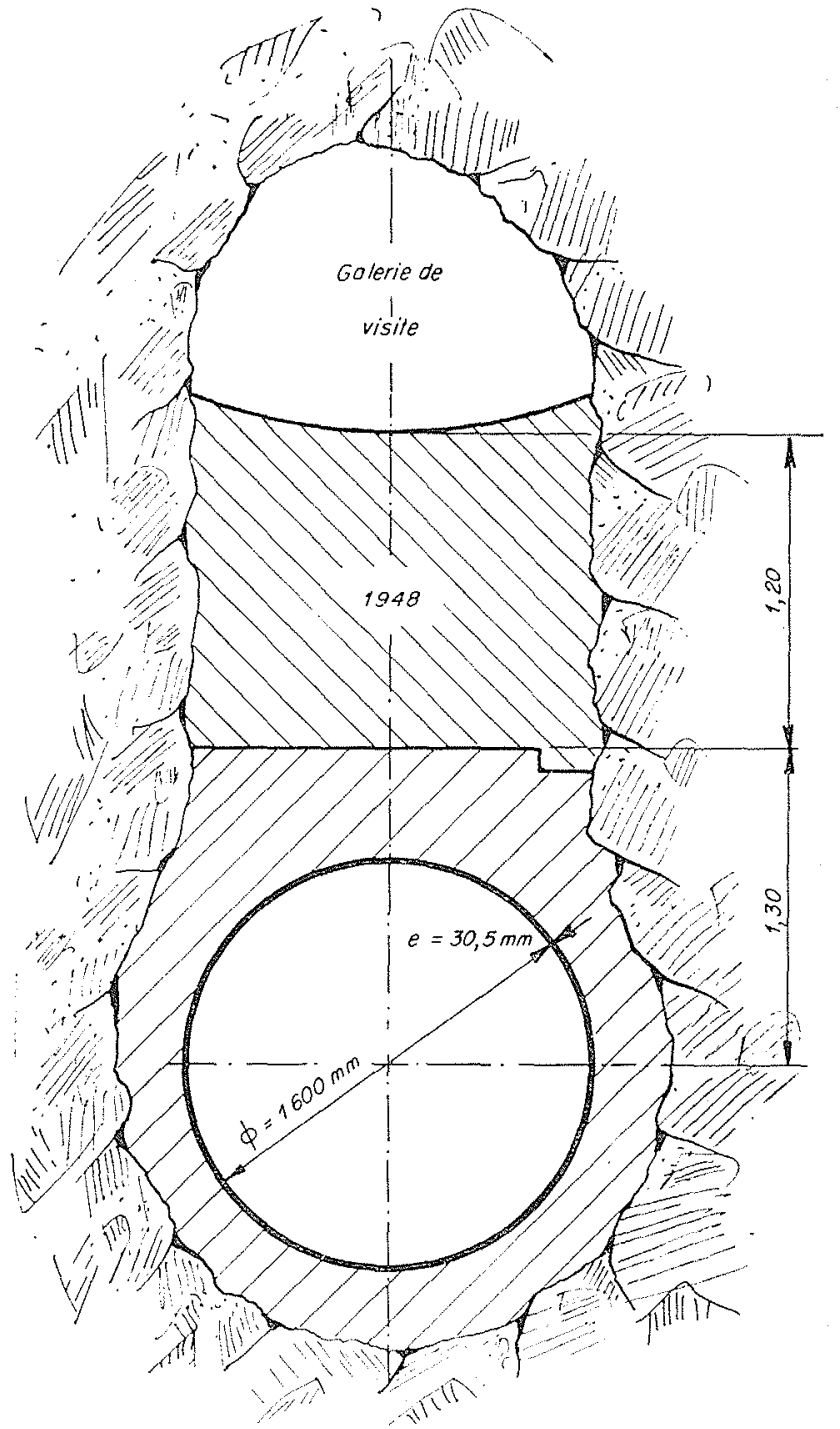

Fig. 22. - Puits de chute de la Gerlos. Partie inférieure.

seulement à un défaut de résistance. Les déchirures suivent bien l'un des rebords du vide de la galerie de drainage ou les deux bords, autant pour la rupture 1 , sur un blindage de $24 \mathrm{~mm}$, que pour les ruptures 2 et 4 . Il apparaît bien que pour ces ruptures la sécurite offerte par les voûtes des galeries de drainage ait été insuffisante (fig. 22). 
D'ailleur's, les efforts de flexion dans la tôle ont été calculés par le professeur Girkmann, en supposant que la voûte de béton ne supportait plus le blindage (fig. 23). Ces efforts semblent assez élevés pour justifier les ruptures constatées. Si la conduite avait été uniformément ten-

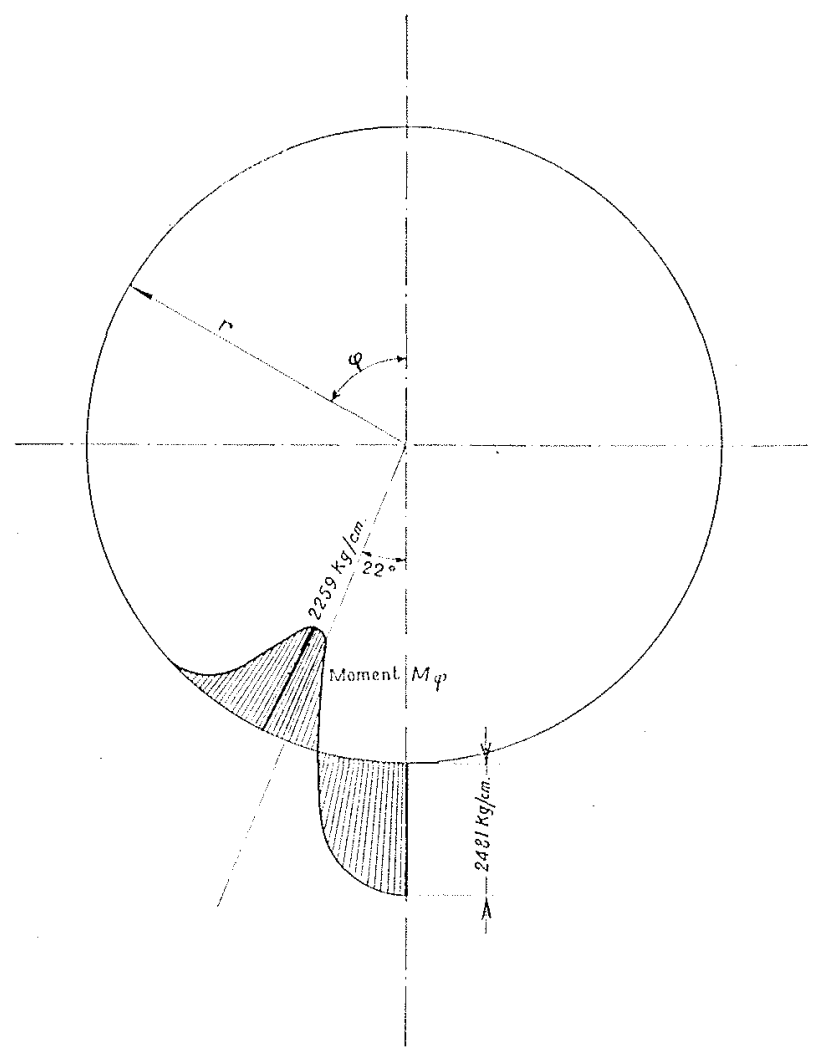

Fig, 23. - FrFont a la Flexion dans te rlindage DF ha Gaterte de la Gerlos.

due, les contraintes n'auraient pas excédé $18 \mathrm{~kg} / \mathrm{mm}^{2}$.

En conclusion, il sera donc prudent de proscrire toutes les causes de dissymétrie au pourtour de la conduite, et notamment les galeries de drainage. Les traversées de roches décomposées recevront un blocage plus épais, très régulier, et s'il le faut armé. L'étanchéité la plus parfaite sera recherchée pour la bonne conservation du rocher. En cas de besoin, des armatures longitudinales devront améliorer la continuité du revêtement.

Il nous faudrait aussi parler des accidents dus aux sous-pressions. Ceux-ci se produisent plutôt au cours des injections de ciment derrière le blindage, ou an cours de vidanges. Les dégâts qu'ils entrainent sont done en général limités. Le serrage du blindage, son étanchéité et la rigidité du béton en éloigneront le danger.

\section{Le coefficient de sécurité et les épreuves des puits de chute}

Pour une conduite en acier posée à l'air libre, le calcul, conduit d'une facon conventionnelle, permet de déterminer une fatigue qui reste inférieure à une certaine proportion de la limite d'élasticité du métal définie par les essais standard. On pent done parler d'un coefficient de sécurité qui sera par exemple 2,5. Ce coefficient tient compte des erreurs possibles et de l'ignorance de l'ingénieur sur certaines données. Le maître de l'œuvre sait bien toutefois que la sécurité sera meilleure si la conduite est construite par un spécialiste qualifié. Si l'ouvrage est réalisé

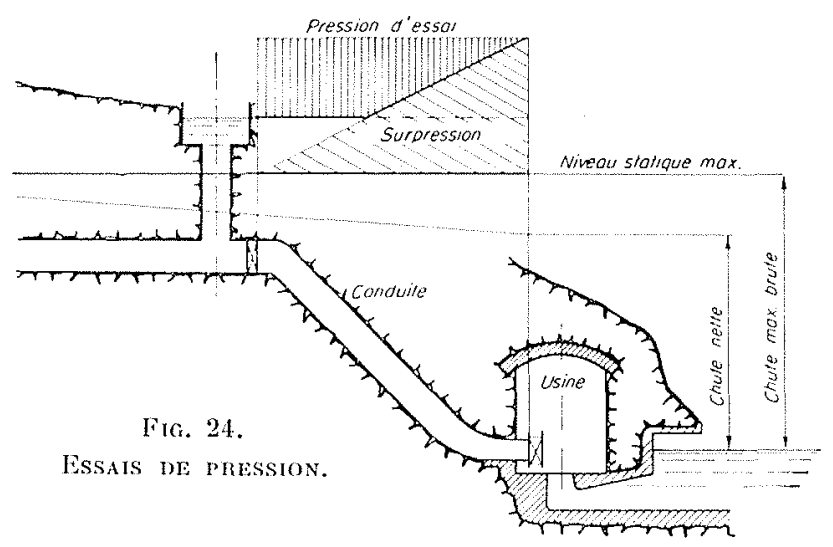

pauvrement, le coefficient de sécurité pourra se montrer illusoire. C'est dire qu'on ne le connaît jamais réellement.

Pour une conduite souterraine, la question se complique du fait qu'interviennent des matériaux dont le contrôle est difficile et les caractéristiques mal définies.

Nous faisons intervenir le terrain, et l'on peut alors répéter avec M. Verdeyen que le coefficient de sécurité est un coefficient d'ignorance dont on ignore et la valeur et la définition. Peutètre la véritable méthode pour assurer la sécurité serait-elle de définir pour chaque élément du calcul, et pour la méthode de calcul ellemême, des fourchettes probables concourant à la composition d'une fourchette résultante, déterminée à la manière d'un calcul d'erreurs. Il faudrait pour que l'ouvrage étudié reste stable que la frontière défavorable de la fourchettc résultante ne soit pas atteinte.

Pour préciser, chaque fourchette pourrait même être remplacée par une courbe de probabilité des erreurs, et les probabilités de rupture pour l'ouvrage devraient être maintenues à un taux défini et extrêmement faible. 
Un tel calcul est difficilement abordable, mais on voit bien que ce ne seront ni les caractéristiques du métal ou du béton qui donneront lieu aux plus grands risques pour un puits de chute, ni même la pression, mais le rocher et les conditions d'exécution du génie eivil de l'ouvrage. C'est sur ces deux points que devra porter avec une sévérité particulière, l'attention du maitre de l'œuvre.

La sécurité sera contrôlée par les épreuves sur l'ouvrage terminé. Une première épreuve est celle de l'étanchéité. Cette épreuve sera faite autant que possible séparément sur chaque partie susceptible de fuites. Par exemple, chaque joint devra être essayé séparément. Les fuites localisées devront rester partout minimes. Les fuites diffuses devront rester très inférieures aux possibilités de drainage naturel par le rocher. Une deuxième épreuve est celle de la pression (fig. 24). Il ne semble pas indispensable de pousser les pressions d'épreuve au delà de la charge maximum effective de service. Ce ne sera même pas souvent possible. D'ailleurs, il est bien d'autres ouvrages auxquels on ne peut appliquer de surcharge. C'est par exemple le cas des barrages.

Par contre, il y aura intérêt à prolonger la durée de charge et à vérifier, au droit des sections douteuses, la stabilité des déformations avec le temps. Les fuites globales de la conduite et son gonflement élastique pourront être mesurés. Il devra être possible de réinjecter les blocages après une première mise en eau.

\section{Conclusion}

Toutefois ce n'est que par une exploitation prolongée que se vérifiera la qualité d'un puits de chute. On peut regretter que de tels ouvrages, extrêmement complexes, se plient mal aux règles simples appliquées couramment à d'autres constructions. Cependant, l'expérience déjà longue que nous en avons, montre que les puits de chute peuvent assurer un long et excellent service si le constructeur a eu le souci de leur durée, s'il n'a troublé que le moins possible le terrain dans son équilibre, s'il a reconstitué cet équilibre, s'il a vérifié méticuleusement la qualité du rocher, et s'il a enfin conduit l'exécution de l'ouvrage avec le maximum de soin.

Les progrès incessants de la technique, et notamment ceux de la mécanique des roches et de la précontrainte, laissent présager un emploi aceru des puits de chute et des conduites souterraines. On peut espérer que ces progrès contribueront de façon décisive au plein succès des réalisations nouvelles.

\section{I S C U S S I O N}

M. le Président remercie M. Taronne et souligne la notion de « l'art de l'Ingénieur » dont M. Talorse met en lumière les multiples aspects dans sá communication.

M. Caquot présente trois remarques :

\& $1^{\circ}$ Au point de vue de la soudure on ne voit pas d'accident important en France, alors qu'en Allemagne, en Belgique et surtout en Amérique ces accidents sont fréquents : il y a quelques jours trois cargos américains se sont cassés en deux lors d'une tempête; d'autre part des Liberty se sont fendus et on a été obligé de les remplacer, tandis qu'aucun bateau, aucun organe français n'a souffert.

« Cela provient de ce qu'en France on ne construit pas avec des matériaux frigiles. Or, actuellement, pour savoir si une tôle est fragile, il faut nécessairement comnaitre sa résilience, et sa résistance à la traction.

"Les constructeurs des souterrains dont vous avez signalé les accidents n'avaient pas pris soin d'exiger pour la fourniture des tôles ces deux paramètres avec des valeurs de non-fragilité.

« Deux exemples :

«a) En fin 1917, j'ai constaté que les vilebrequins cassaient dans les moteurs faute d'une résilience suffisante. Il a suffi de l'exiger pour faire cesser les ruptures;

«b) Plus tard, au pont de Blanville, les barres de construction du pont tombant de $80 \mathrm{~cm}$ se cassaient toutes. Tous les essais du cahier des charges étaient bons, mais il n'était pas prévu de mesure de résilience. « Done il est nécessaire de ne pas utiliser de tôles sans les deux paramètres correspondant à la courbe intrinsèque des aciers : profondeur et largeur, ou résilience ct traction. Si les tôles ne sont pas fragiles et si les dispositions sont correctes, il n'y aura pas d'accident.

« $2^{\circ}$ J'ai prévu les revêtements du Sautet, de Cordéac et de Moux et je n'ai pas eu de déboires bien que la roche fût mauvaise à Cordéac; ceci parce que lorsque l'on perce une galerie dans une roche, quel que soit le soin apporté au percement de la galerie, la roche a dépassé la limite élastique : si vous la calculez d'après les formules de Laine, vous trouvez pour une pression hydrostatique $P$ du terrain en supposant un système homogène après percement une compression simple de $2 P$ à la paroi. Or, la plupart des roches ne peuvent pas supporter cette pression sans se plastifier à la paroi C'est cette zone que vous avez indiquée très justement tout à l'heure comme devant être injectée pour rétablir sa résistance, et j'ai tenu compte de sa plastification.

"Pour préciser ma pensée, je dirai par exemple qu'il est impossible de connaître en souterrain sur le front d'attaque la nature de la roche en place, parce que le percement détermine des glissements dans les surfaces libérées. Si le projet tient compte de ces faits, il peut, en toute sécurité, prendre les mesures nécessaires pour 
équilibrer sans fuite des pressions de l'ordre de $2.000 \mathrm{~m}$ d'eau. Je crois que nous sommes plus avancés dans ces questions scientifiques que les ingénieurs américains et il serait intéressant de discuter scientifiruement ces problèmes. $»$

M. le Président remercie M. Canoot de ces remarques intéressantes.

M. Hupner se demande si les modes habituels de perforation des galeries n'aggravent pas les fissurations de la roche et s'il ne serait pas possible d'éviter ou de réduire celles-ci par l'emploi de perceuses rotatives, analogues à celles utilisées pour le perement de puits verticaux, mais encore non appliquées en France au creusement de galeries sub-horizontales.

M. Caquot rappelle que ces machines ont été étudiées en France en 1888 en vue du percement du tunnel sous la Manche, et decrites dans le catalogue de l'Exposition de 1889. M. Caevot estime que la mise au concon's de leur application au pereement des galeries d'aménage. ment hydro-électriques dans les roches demi-dures permetrait à Electricité de France une sensible diminution de prix de ces importants ouvrages.

M. Rementerras présente les trois observations suirantes :

$1^{\circ}$ Des essais ont été cfectués récemment au Portugal (Laboratorio de Engenheria Civil de Lisbonne) pour mesurce le module d'élasticité du granit en galerie, avant et après injection de la roche. Les pressions étaient engendrées par un vérin placé suivant un diamètre horizontal de la galerie et transmises à deux portions opposées du parement de celle-ci par l'intermédiaire do «coussins》 remplis d'huile.

Pour la roche brute de perforation, le diagramme «Effort-Déformation » comprend deux parties ì peu près rectilignes; la première donne un module d'élasticité assez faible qui correspond à la période dite do « serrage» qui se place an début de la « mise en pression $\gg$; la deuxième, qui lui fait suite, conduit à un module beaucoup plus élevé se rapprochant de celui que l'on mesurerait au laboratoire sur éprouvettes.

La portion de galerie soumise à l'essai ci-dessus ayant été injectée de ciment, derrière un revêtement provisoire qui est ensuite démoli, on lelève à nouveau le diagramme «Effort-Déformation 》; celui-ci ne comprend alors - dans la limite des pressions réalisées - qu'une seule droite dont la pente correspond au module d'élasticité élevé trouvé dans la première expérience au-delá de la « zone de serrage ».

Il réulterait donc de cette expérience que la roche injecté de ciment présente sensiblement les unches proprietes mécaniques que la roche compacte non diaclasée dans lacuelle on taille dordinate les eprourettes soumises aux essais de laboratoire.

2" Il est possible de contrôler globalement la répartition des contraintes entre la tôle, le revêtement bétonné et le rocher dans un tronçon de salerie bindée, en mesurant la célérité, à la traversée de ce troncon rempli d'ean, de l'onde de surpression créée par un coup de bélier de fable amplitude. Cette célérité dépend en effet, au premier" chef, de la déformation de la paroi de la galerie sous laction de la pression. La méthode a été ulilisce avee suecès en Italic (1). Elle présente l'ineonvénient de ne pouvoir deceler un point faible localisé, car elle détermine en fait la déformation moyenne, en fonction de la pression, d'un secteur de galerie qui doit cire assez long pour que la mesure garde une précision suffisante. Telle quelle, clle peut pourtant etre intéressante comme méthode de réception et de contrôle.

$3^{\circ}$ Dans le calcul de la répartition des contraintes entre la tôle, le béton et le rocher, il y aurait lieu de tenir compte des phénomènes de fluage que présentent ces deux derniers matériaux; des essais, tels que celui envisagé au paragraphe $2^{\circ}$, échelonnés sur les premières années de service de la galerie, pourraient donner quelques indications sur l'influence du fluage.

M. le Président remereie M. Remenibras de son intervention et souligne que la conférence faite par M. 'TaLome fait honneur non seulement au conférencier mais aux techniciens d'E.D.F. en général.

(1) Voir E. Gracr: Contribution à l'étude du comportement des conduites forces noyees dans la roche. Elettrotecnica, $\mathrm{n}^{\circ}$ ll, no vemlire $19+9$, pp. 546.550

\section{1" CONGRES DE L'ASSOCIATYON FRANCAISE POUR L'AVANCEMENT DES SCIENCES}

Cannes : 22-27 septembre 1952

Le $71^{\circ}$ Congrès de $1^{\prime}$ Association Française pour l'Avancement des Sciences se tiendra ì Cannes du 2. an 27 septembre 1952, sous la présidence de M. Jean Cabannes, Membre de l'Institul, Doyen d'honneur de la Faculté des Sciences.

Il comportera plusieurs séances de travail par section dans le Palais des Festivals, et des exeursions aux îles de Lérins, à Fréjus, Menton, Monaco, etc.

Un programme provisoire est déposé au secrétariat de la S.H.F. à la disposition de ses membres.

Pour tous renseignements complementaires, s'adresser au siège de l'Association Française pour l'Avancement des Sciences, 28, rue Serpente, Paris $\left(6^{\circ}\right)$. 